\title{
Higher-order topology and corner triplon excitations in two-dimensional quantum spin-dimer models
}

\author{
Arijit Haldar, ${ }^{*}$ Geremia Massarelli, ${ }^{\dagger}$ and Arun Paramekanti ${ }^{\ddagger}$ \\ Department of Physics, University of Toronto, 60 St. George Street, Toronto, Ontario M5S 1A7 Canada
}

(Dated: November 23, 2021)

\begin{abstract}
The concept of free fermion topology has been generalized to $d$-dimensional phases that exhibit $(d-n)$-dimensional boundary modes, such as zero-dimensional (0D) corner excitations. Motivated by recent extensions of these ideas to magnetic systems, we consider $2 \mathrm{D}$ quantum paramagnets formed by interacting spin dimers with dispersive triplet excitations. We propose two examples of such dimer models, where the spin-gapped bosonic triplon excitations are shown to host bands with nontrivial higher-order topology. We demonstrate this using real-space Bogoliubov-de Gennes calculations that reveal the existence of near-mid-bandgap corner triplon modes as a signature of higher-order bulk topology. We provide an understanding of the higher-order topology in these systems via a computation of bulk topological invariants as well as the construction of edge theories, and study their phase transitions as we tune parameters in the model Hamiltonians. We also discuss possible experimental approaches for detecting the emergent corner triplon modes.
\end{abstract}

\section{INTRODUCTION}

Higher-order topological (HOT) systems have emerged as the next step in understanding the rapidly growing family of topological phases in condensed matter physics. As a natural generalization of conventional or first-order topology, an $n^{\text {th }}$-order topological system in $d$-dimensions hosts protected gapless modes on any of its $(d-n)$ dimensional boundaries. For instance, a three-dimensional (3D) HOT system exhibiting second-order topology hosts protected hinge modes, while one exhibiting third-order topology hosts protected corner modes. Similarly, a 2D HOT system showing second-order topology hosts localized corner modes. First discovered in the context of electric-multipolar insulators [1] in fermionic systems with quantized moments, the list of HOT systems was quickly expanded to include chiral and helical HOT insulators [2]. The core idea behind fermionic HOT insulators has recently been generalized, either theoretically or experimentally, to alternate platforms such as mechanical [3], electrical [4], microwave [5], photonic [6], non-Hermitian [7], and superconducting [8-14] systems.

In addition to these platforms, proposals to observe HOT physics in quantum spin systems [15-17] and solvable spin liquid models [18-20] have also been put forward. While investigations of HOT phases in these spin systems have mainly focused on ground-state physics, their generalization to excited states is relatively less explored. A few recent studies have tried to bridge this gap by proposing spin models with magnetically ordered ground states. Although the ground states are topologically trivial, the HOT phases in these magnetic systems emerge as hinge [21, 22] or corner [23-25] magnon excitations. In this paper, we explore the opposite regime; i.e., we study

\footnotetext{
*arijit.haldar@utoronto.ca

† gmassare@physics.utoronto.ca

$\ddagger$ arun.paramekanti@utoronto.ca
}

spin models without any magnetic order. We demonstrate that even spin-gapped quantum paramagnets, formed by coupled spin dimers, can host excitations that show HOT physics.

Spin dimers arise naturally in Mott insulators where certain adjacent pairs of sites are coupled by strong exchange interactions, with weaker inter-dimer exchange couplings [26-36]. They can also arise in geometrically frustrated magnets which may exhibit spontaneous dimerization and lattice symmetry breaking via formation of valence bond crystals [37, 38]. On a single dimer with strong antiferromagnetic intra-dimer Heisenberg exchange, the ground state is a singlet, and the gapped excited states form triplets with total spin $S=1$. Weaker inter-dimer couplings in the crystal induce hopping and pairing terms for the triplet excitations. Such singlet quantum paramagnets thus exhibit a spectrum of gapped and dispersive triplet excitations called triplons.

With full spin-rotational symmetry, the triplons exhibit three decoupled and degenerate bands, corresponding to the three distinct spin states. However, in the presence of spin-orbit coupling (SOC) or an applied magnetic field, the different spin states get coupled and this band degeneracy is broken. It has been shown that the resulting triplon bands can exhibit non-trivial first order topology, with nonzero Chern numbers and a significant thermal Hall effect $[39,40]$. In our work, we show that such quantum paramagnets can also exhibit HOT phases and triplon corner-mode excitations that arise as near-mid-bandgap states in their triplet excitation spectrum.

This paper is organized as follows: In Sec. II, we formally define the two spin models which exhibit the HOT triplon phases. In Sec. III, we develop the effective theory of triplon excitations for the spin models. The emergent triplon phases for the models, including the HOT phases, are discussed in Sec. IV, and the symmetries that protect the HOT phases are discussed in Sec. V. We characterize the topological triplon phases using topological invariants such as the Wannier-sector polarizations in Sec. VI. Finally, we develop the effective edge theory for the models 
in Sec. VII, allowing us to show that the corner modes arise at the intersection between two edges. We conclude in Sec. VIII with a summary of the work and a discussion on possible experimental realization. Additional details about various results are provided in the appendices, and are referred to in the main text.

\section{MODEL HAMILTONIANS}

We consider an array of spin dimers described by the Hamiltonian

$$
\hat{H}_{\mathrm{D}}=J_{\mathrm{D}} \sum_{\mathbf{r}} \mathbf{S}_{\mathbf{r} T} \cdot \mathbf{S}_{\mathbf{r} B}
$$

with $J_{\mathrm{D}}>0$, where spins live on a bilayer square lattice. The square-lattice sites are labeled by $\mathbf{r}$, and $T, B$ refer to 'top' and 'bottom' spins on the bilayer which form the dimer at $\mathbf{r}$. The unique ground state of the Hamiltonian $\hat{H}_{\mathrm{D}}$ is a direct product of interlayer dimer singlets at each site $\mathbf{r}$. The lowest energy excitations correspond to creating a triplet on any one of the dimers with the spin gap energy $J_{\mathrm{D}}$. We next introduce two distinct models of inter-dimer exchange interactions and demonstrate that the resulting quantum paramagnets host HOT triplon excitations.

\section{A. Model I: The tBBH model}

Our first model, an XXZ model of inter-dimer couplings, is directly inspired by the $\mathrm{BBH}$ model of spinless fermions [1]. Hence, we name this the tBBH model, for "triplon BBH model". To define the model, we group four dimers together into a unit cell as shown in Figs. 1(a) and 1(b). The total spin Hamiltonian is given by

$$
\begin{gathered}
\hat{H}_{\mathrm{tBBH}}^{\mathrm{spin}}=\hat{H}_{\mathrm{D}}+\hat{H}_{I} \\
\hat{H}_{I}=\sum_{\left\langle\mathbf{r} \mathbf{r}^{\prime}\right\rangle, \ell} J_{\mathbf{r}, \mathbf{r}^{\prime}}\left[\zeta_{\mathbf{r}, \mathbf{r}^{\prime}}\left(S_{\mathbf{r} \ell}^{x} S_{\mathbf{r}^{\prime} \ell}^{x}+S_{\mathbf{r} \ell}^{y} S_{\mathbf{r}^{\prime} \ell}^{y}\right)+S_{\mathbf{r} \ell}^{z} S_{\mathbf{r}^{\prime} \ell}^{z}\right]
\end{gathered}
$$

where $\ell=T, B$ labels the layers. The inter-dimer exchange $J_{\mathbf{r}, \mathbf{r}^{\prime}}$ takes on values $J_{1}$ and $J_{2}$ for neighboring sites which are in the same unit cell or in adjacent unit cells, respectively. The coefficient $\zeta_{\mathbf{r}, \mathbf{r}^{\prime}}$ defines the sign of the XY exchange, taking on values $+1(-1)$ on the solid (dashed) bonds as shown in Fig. 1(b); as we show below, this sign choice leads to the triplons sensing a $\pi$-flux ${ }^{1}$ in each elementary square plaquette. For this Hamiltonian, only two of the three triplons $(x, y)$ will form HOT bands, as we discuss later, while the $z$-triplon remains in

\footnotetext{
1 This is not a physical electromagnetic flux, but rather the phase accumulated by the triplon wavefunction as the triplon hops around a single plaquette.
}

a topologically trivial phase. The model may be considered an example of Su-Schrieffer-Heeger (SSH) [41] spin ladders running along $\hat{x}$ which are coupled by frustrated inter-ladder interactions; a recent experimental example of such an SSH spin ladder is discussed in Ref. 42.

\section{B. Model II: The tSOC model}

To get higher-order topology to emerge in 2D fermionic systems, it has been shown that we need at least four degrees of freedom per unit cell [1]. Heuristically, the polarizations defining the first-order topologies of two bands can then potentially cancel with each other, allowing for the higher order quadrupole moment to emerge as the proper characterization of the nontrivial band topology of this band pair. In our first model, the different triplon flavors are decoupled, and the relevant degrees of freedom arise from having four dimers in each unit cell. In our second model below, we instead include SOC that mixes two of the three triplon flavors, which permits a smaller unit cell consisting of two dimers. Therefore, we refer to this model as the tSOC model, short for "triplons with spin-orbit coupling".

The model is constructed (see Figs. 1(c) and (d)) as an array of 1D ladders along $y$, each ladder being described by a model which hosts triplon bands with nontrivial first-order topology as shown by Joshi and Schnyder [43]. In the transverse $(x)$ direction, the chains are coupled by alternating Heisenberg couplings which realize an $\mathrm{SSH}$ spin model. The Hamiltonian for the tSOC model is given by

$$
\begin{gathered}
\hat{H}_{\mathrm{tSOC}}^{\mathrm{spin}}=\hat{H}_{\mathrm{D}}+\hat{H}_{I I} \\
\hat{H}_{I I}=\sum_{\left\langle\mathbf{r} \mathbf{r}^{\prime}\right\rangle, \ell} J_{\mathbf{r}, \mathbf{r}^{\prime}} \mathbf{S}_{\mathbf{r} \ell} \cdot \mathbf{S}_{\mathbf{r}^{\prime} \ell}+D \sum_{\left\langle\mathbf{r}^{\prime}\right\rangle_{y}, \ell} \zeta_{\mathbf{r}, \mathbf{r}^{\prime}}\left(S_{\mathbf{r} \ell}^{x} S_{\mathbf{r}+y \ell}^{y}-S_{\mathbf{r} \ell}^{y} S_{\mathbf{r}+y \ell}^{x}\right) \\
+\Gamma \sum_{\left\langle\mathbf{r} \mathbf{r}^{\prime}\right\rangle_{y}, \ell} \zeta_{\mathbf{r}, \mathbf{r}^{\prime}}\left(S_{\mathbf{r} \ell}^{x} S_{\mathbf{r}+y \ell}^{y}+S_{\mathbf{r} \ell}^{y} S_{\mathbf{r}+y \ell}^{x}\right)
\end{gathered}
$$

Along the $x$ direction, $J_{\mathbf{r}, \mathbf{r}^{\prime}}$ takes on two distinct values $J_{1}$ and $J_{2}$ for intra-unit-cell nearest-neighbor spins and inter-unit-cell nearest neighbor spins, respectively (see Fig. $1(\mathrm{~d}))$. Along the $y$ direction, $J_{\mathbf{r}, \mathbf{r}^{\prime}}=J$ for nearestneighbor spins. The $D$ and $\Gamma$ terms represent additional off-diagonal exchange interactions - the antisymmetric Dzyaloshinskii-Moriya (DM) coupling $D$ and its symmetric counterpart $\Gamma$. As in the previous model, the coefficient $\zeta_{\mathbf{r}, \mathbf{r}^{\prime}}$ takes on values $+1(-1)$ on the solid (dashed) bonds as shown in Fig. 1(d), so that the DM and $\Gamma$ interactions alternate in sign from one $y$-direction ladder to the next.

\section{TRIPLON EXCITATIONS}

The models defined in Sec. II possess a trivial quantum paramagnetic ground state when the inter-dimer 
(a)

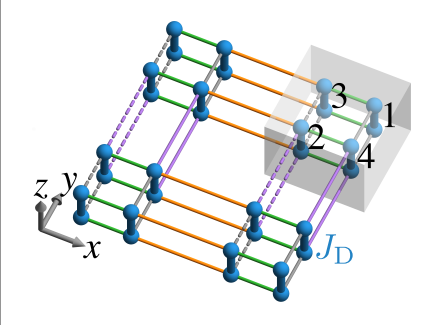

(b)

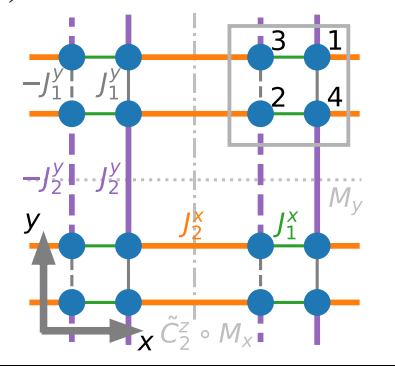

(c)

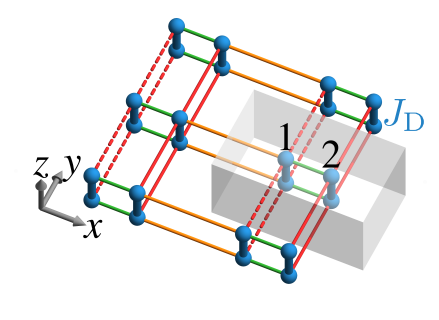

(d)

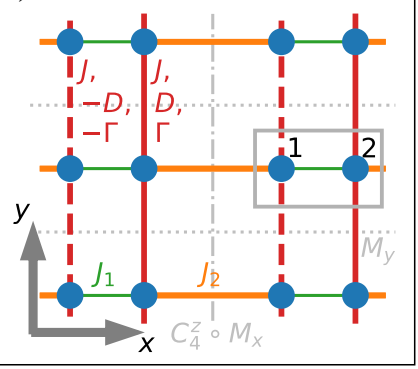

FIG. 1. Models: (a) The lattice of spin dimers hosting the triplon-BBH model (tBBH), made up of a top (T) and a bottom (B) layer. The unit cell is composed of four dimers numbered 1 to 4. (b) A top-down view of the lattice in (a); thin (thick) horizontal bonds have $J_{1}^{x}\left(J_{2}^{x}\right)$ Heisenberg interactions, and thin (thick) vertical bonds have interactions proportional to $J_{1}^{y}$ $\left(J_{2}^{y}\right)$, respectively. The sign of the interactions on the vertical bonds alternates as we move along the $x$-direction. The dashed vertical lines have $-J_{1,2}^{y}$ and the solid vertical lines have $+J_{1,2}^{y}$ interactions. (c) The bilayer spin dimer lattice used to realize a HOT phase in the tSOC model for triplons with spin-orbit coupling (SOC). The model has a reduced unit cell (compared to (a)) consisting of two dimers numbered 1 and 2. (d) A top-down view of the lattice in (c); horizontal bonds have the same configuration as in (b) with thin (thick) bonds having $J_{1}\left(J_{2}\right)$ Heisenberg interactions. The vertical bonds have DM $(D)$ and Gamma $(\Gamma)$ interactions that introduce SOC into the model, in addition to a small Heisenberg interaction $J$. The direction of DM and Gamma interactions alternates along the $x$ direction, with dashed lines having DM and Gamma interactions pointing along the $-\hat{z}$ direction, and solid lines in the $+\hat{z}$ direction. Mirror axes described in Sec. V are shown in the top-down views in dotted and dashed-dotted lines.

exchanges are much weaker than $J_{\mathrm{D}}$ (Eq. 1). In order to describe the excitations above the singlet ground state, we use the bond operator formalism [44, 45], which maps the triplets and singlet states of each dimer to bosons as follows

$$
\begin{aligned}
t_{x}^{\dagger}|0\rangle & =\mathrm{i}(|\uparrow \uparrow\rangle-|\downarrow \downarrow\rangle) / \sqrt{2} \\
t_{y}^{\dagger}|0\rangle & =\quad(|\uparrow \uparrow\rangle+|\downarrow \downarrow\rangle) / \sqrt{2} \\
t_{z}^{\dagger}|0\rangle & =-\mathrm{i}(|\uparrow \downarrow\rangle+|\downarrow \uparrow\rangle) / \sqrt{2} \\
s^{\dagger}|0\rangle & =\quad(|\uparrow \downarrow\rangle-|\downarrow \uparrow\rangle) / \sqrt{2} .
\end{aligned}
$$

Here we have labeled the three triplet flavors [46] as $x, y, z$. The bosonic operators $t_{\alpha}^{\dagger}$ (with $\alpha \in\{x, y, z\}$ ) create triplons from the vacuum state $|0\rangle$, and $s^{\dagger}$ does the same for a singlet. Since each dimer has exactly one singlet or triplet, we must impose the constraint $s^{\dagger} s+\sum_{\alpha} t_{\alpha}^{\dagger} t_{\alpha}=1$, where $s^{\dagger} s$ and $t_{\alpha}^{\dagger} t_{\alpha}$ gives the number densities for singlets and $\alpha$ triplets respectively. With this constraint, the spin operators on the two sites at a dimer can be represented in terms of the bosons as follows:

$$
\begin{aligned}
& S_{\mathbf{r} T}^{\alpha}=\frac{1}{2}\left(+\mathrm{i} t_{\mathbf{r} \alpha}^{\dagger} s_{\mathbf{r}}-\mathrm{i} s_{\mathbf{r}}^{\dagger} t_{\mathbf{r} \alpha}-\mathrm{i} \varepsilon_{\alpha \beta \gamma} t_{\mathbf{r} \beta}^{\dagger} t_{\mathbf{r} \gamma}\right) \\
& S_{\mathbf{r} B}^{\alpha}=\frac{1}{2}\left(-\mathrm{i} t_{\mathbf{r} \alpha}^{\dagger} s_{\mathbf{r}}+\mathrm{i} s_{\mathbf{r}}^{\dagger} t_{\mathbf{r} \alpha}-\mathrm{i} \varepsilon_{\alpha \beta \gamma} t_{\mathbf{r} \beta}^{\dagger} t_{\mathbf{r} \gamma}\right)
\end{aligned}
$$

Deep in the paramagnetic phase, the singlet density is approximately 1 . Throughout this paper we will work in this limit, and therefore set $s$ and $s^{\dagger}$ to the c-number 1 [44]. Furthermore, we shall only keep terms that are bilinear in the triplon operators $t_{\alpha}^{\dagger}$ and $t_{\alpha}[39,43,44]$.

In deriving the effective triplon Hamiltonians below to describe the spin-gapped excitations, we relabel the sites $\mathbf{r}:=(\mathbf{R}, a)$ where $\mathbf{R}$ denotes unit cell and $a$ stands for the sublattice index. For the tBBH model (model I), the sublattice index $a \in\{1,2,3,4\}$, while for the tSOC model (model II), $a \in\{1,2\}$. For compactness of notation, we define two-component Nambu spinors $\phi_{\mathbf{R}, a \alpha}^{\dagger}=\left[t_{\mathbf{R}, a \alpha}^{\dagger} t_{\mathbf{R}, a \alpha}\right]$. For both models, the $z$ triplon remains decoupled with topologically trivial bands. We will henceforth focus only on those sectors which exhibit nontrivial topology; with this understanding, the triplon label $\alpha$ will be used to denote only $\alpha \in\{x, y\}$.

a. Model I ( $t B B H$ model) In the first model, the different triplet flavor states $\alpha$ are decoupled at quadratic order due to the absence of SOC and Zeeman fields. We construct the eight-component bosonic Nambu vector $\Phi_{\mathbf{R} \alpha}^{\dagger}=\left[\phi_{\mathbf{R}, 1 \alpha}^{\dagger} \phi_{\mathbf{R}, 2 \alpha}^{\dagger} \phi_{\mathbf{R}, 3 \alpha}^{\dagger} \phi_{\mathbf{R}, 4 \alpha}^{\dagger}\right]$. The real-space triplon Hamiltonian, obtained by expressing the spin operators in Eq. 2 using the relations in Eq. 5, is then given by

$$
\hat{H}_{\mathrm{tBBH}}=\frac{1}{2} \sum_{\mathbf{R}, \boldsymbol{\delta}, \alpha} \Phi_{\mathbf{R}+\delta \alpha}^{\dagger} \mathbb{M}_{\boldsymbol{\delta}} \Phi_{\mathbf{R} \alpha}
$$

with the coupling matrices

$$
\begin{aligned}
\mathbb{M}_{0} & =J_{\mathrm{D}} \sigma_{0} \eta_{0} \tau_{0}+\left(\frac{J_{1}}{2} \sigma_{1} \eta_{0}-\frac{J_{1}}{2} \sigma_{2} \eta_{2}\right)\left(\tau_{0}-\tau_{1}\right) \\
\mathbb{M}_{+\hat{x}} & =\mathbb{M}_{-\hat{x}}^{\dagger}=\frac{J_{2}}{4}\left(\sigma_{1} \eta_{0}-\mathrm{i} \sigma_{2} \eta_{3}\right)\left(\tau_{0}-\tau_{1}\right) \\
\mathbb{M}_{+\hat{y}} & =\mathbb{M}_{-\hat{y}}^{\dagger}=-\frac{J_{2}}{4} \mathrm{i} \sigma_{2}\left(\eta_{1}-\mathrm{i} \eta_{2}\right)\left(\tau_{0}-\tau_{1}\right) .
\end{aligned}
$$

Here, $\sigma_{i}, \eta_{i}$, and $\tau_{i}$ correspond to sets of Pauli matrices with Kronecker product implied between them. Specifically, $\tau_{i}$ acts in Nambu (particle-hole) space, while $\sigma_{i}$ and $\eta_{i}$ act in the space of sublattices $a \in\{1,2,3,4\}$. In terms of the $\left(\sigma_{3}, \eta_{3}\right)$ eigenvalues, the sublattices $a=(1,2,3,4)$ 
correspond respectively to $(++,+-,-+,--)$. Fouriertransforming this yields the momentum-space Hamiltonian $\hat{H}_{\mathrm{tBBH}}=\frac{1}{2} \sum_{\mathbf{k} \alpha} \Phi_{\mathbf{k} \alpha}^{\dagger} H_{\mathbf{k}}^{\mathrm{tBBH}} \Phi_{\mathbf{k} \alpha}{ }^{2}$, where $H_{\mathbf{k}}^{\mathrm{tBBH}}$ is an $8 \times 8$ matrix independent of $\alpha$, given by

$$
\begin{aligned}
H_{\mathbf{k}}^{\mathrm{tBBH}}= & J_{\mathrm{D}} \sigma_{0} \eta_{0} \tau_{0}+\left(\frac{J_{1}}{2} \sigma_{1} \eta_{0}-\frac{J_{1}}{2} \sigma_{2} \eta_{2}\right)\left(\tau_{0}-\tau_{1}\right) \\
+ & \frac{J_{2}}{2}\left(\cos k_{x} \sigma_{1} \eta_{0}-\sin k_{x} \sigma_{2} \eta_{3}\right)\left(\tau_{0}-\tau_{1}\right) \\
& \quad-\frac{J_{2}}{2} \sigma_{2}\left(\cos k_{y} \eta_{2}+\sin k_{y} \eta_{1}\right)\left(\tau_{0}-\tau_{1}\right) .
\end{aligned}
$$

b. Model II (tSOC model) In the second model, SOC couples the $x$ and $y$ triplon sectors. We define the eight-component bosonic Nambu vector $\Psi_{\mathbf{R}}^{\dagger}=$ $\left[\phi_{\mathbf{R}, 1 x}^{\dagger} \phi_{\mathbf{R}, 1 y}^{\dagger} \phi_{\mathbf{R}, 2 x}^{\dagger} \phi_{\mathbf{R}, 2 y}^{\dagger}\right]$, in terms of which the triplon Hamiltonian is given by

$$
\hat{H}_{\mathrm{tSOC}}=\frac{1}{2} \sum_{\mathbf{R}, \boldsymbol{\delta}} \Psi_{\mathbf{R}+\delta}^{\dagger} \mathbb{M}_{\delta} \Psi_{\mathbf{R}}
$$

with the coupling matrices given by

$$
\begin{aligned}
\mathbb{M}_{0} & =J_{\mathrm{D}} \sigma_{0} \eta_{0} \tau_{0}+\frac{J_{1}}{2} \sigma_{1} \eta_{0}\left(\tau_{0}-\tau_{1}\right) \\
\mathbb{M}_{+\hat{x}} & =\mathbb{M}_{-\hat{x}}^{\dagger}=\frac{J_{2}}{4}\left(\sigma_{1}+\mathrm{i} \sigma_{2}\right) \eta_{0}\left(\tau_{0}-\tau_{1}\right) \\
\mathbb{M}_{+\hat{y}} & =\mathbb{M}_{-\hat{y}}^{\dagger}=\frac{J \sigma_{0} \eta_{0}+\sigma_{3}\left(\Gamma \eta_{1}+\mathrm{i} D \eta_{2}\right)}{2}\left(\tau_{0}-\tau_{1}\right) .
\end{aligned}
$$

While $\tau_{i}$ Pauli matrices again act in Nambu (particlehole) space, here the $\sigma_{i}$ and $\eta_{i}$ Pauli matrices respectively act on the sublattice index $a \in(1,2)$ and triplon flavor index $\alpha \in(x, y)$. Thus the two triplon flavors here take on the role of the two additional sublattices in model I. Fourier-transforming leads to the $8 \times 8$ Bloch Hamiltonian:

$$
\begin{aligned}
& H_{\mathbf{k}}^{\mathrm{tSOC}}= J_{\mathrm{D}} \sigma_{0} \eta_{0} \tau_{0}+\frac{J_{1}}{2} \sigma_{1} \eta_{0}\left(\tau_{0}-\tau_{1}\right) \\
&+\frac{J_{2}}{2}\left(\cos k_{x} \sigma_{1}+\sin k_{x} \sigma_{2}\right) \eta_{0}\left(\tau_{0}-\tau_{1}\right) \\
&+\left(\left(J \sigma_{0} \eta_{0}+\Gamma \sigma_{3} \eta_{1}\right) \cos k_{y}+D \sin k_{y} \sigma_{3} \eta_{2}\right)\left(\tau_{0}-\tau_{1}\right) .
\end{aligned}
$$

c. Diagonalization Triplon Hamiltonians like $\hat{H}_{\mathrm{tBBH}}$ and $\hat{H}_{\mathrm{tSOC}}$ are in general bosonic Bogoliubov-de Gennes (BdG) Hamiltonians [44]. We briefly review the process for solving such Hamiltonians: writing $\hat{H}=\frac{1}{2} \sum_{\mathbf{k}} \Phi_{\mathbf{k}}^{\dagger} H_{\mathbf{k}} \Phi_{\mathbf{k}}$, $\hat{H}$ is diagonalized via a paraunitary Bogoliubov transformation $\Phi_{\mathbf{k}}=T_{\mathbf{k}} \Gamma_{\mathbf{k}}$, where the matrices $T_{\mathbf{k}}$ satisfy $T_{\mathbf{k}}^{\dagger} \tau_{3} T_{\mathbf{k}}=\tau_{3}$, where $\tau_{3}$, short for $1_{n} \otimes \tau_{3}$, is the third Pauli matrix in Nambu space [47-50]. Provided $H_{\mathbf{k}}$ is

\footnotetext{
2 The redundancy intrinsic to the Nambu formalism is eliminated by choosing coefficient matrices to be particle-hole symmetric. In momentum space, this constraint takes the form $H_{\mathbf{k}}=\tau_{1} H_{-\mathbf{k}}^{*} \tau_{1}$
}

positive definite, such a transformation $T_{\mathbf{k}}$ exists, and the eigenvectors of $\tau_{3} H_{\mathbf{k}}$ can be chosen to construct the columns of $T_{\mathbf{k}}$. The BdG structure of the Hamiltonian guarantees that half the eigenvalues of $\tau_{3} H_{\mathbf{k}}$ are positive (particle like) and the other half is negative (hole like). The positive eigenvalues give the energies for the bosonic modes at a given momentum $\mathbf{k}$. The diagonalization procedure for a position-space BdG Hamiltonian can also be performed in a similar fashion.

\section{HOT PHASE AND TOPOLOGICAL TRANSITION}

A characteristic feature of a 2D HOT phase is the existence of topological corner modes. To check for these corner modes, we solve the triplon real space Hamiltonians in Eq. 6 and Eq. 8 on a finite geometry with open boundary conditions (OBC) in the $x$ and $y$ directions.

For the first model, given by $\hat{H}_{\mathrm{tBBH}}$ (Eq. 6), we fix parameters $J_{1} / J_{\mathrm{D}}=0.02$ and $J_{2} / J_{\mathrm{D}}=0.2$ and plot the energy spectrum for the $t_{x}$ triplons in Fig. 2(a). It shows an energy bandgap with four modes near the middle of the bandgap $\left(E / J_{\mathrm{D}} \approx 1.0\right)$, right about where we would expect to find the corner-mode energies. We use the term "near" to specify the location of these energies, as we find the spectrum is not symmetric about $E / J_{\mathrm{D}}=1.0$ for a finite value of $J_{\mathrm{D}}$. However, the spectrum becomes symmetric asymptotically as $J_{\mathrm{D}} \rightarrow \infty$, and the energies corresponding to these four modes shift to exactly the middle of the bandgap. To verify that these are corner modes, we plot the real-space triplon density averaged over all four of these modes and overlay it over our finite lattice geometry; see Fig. 2(c). Indeed, we see that the density peaks at the corners, confirming the existence of corner modes. In addition to the corner modes, we find edge modes whose energies lie inside the bandgap but are not pinned to the energy $E \approx J_{\mathrm{D}}$ inside the bandgap (see Fig. 2(a)) ${ }^{3}$; such states also exist in the fermionic BBH model [1]. We show the density profile of one of these edge modes in the bottom inset of Fig. 2(a).

To check for corner modes in the second model, we use parameter values $J_{1} / J_{\mathrm{D}}=0.1, J_{2} / J_{\mathrm{D}}=0.2, D / J_{\mathrm{D}}=0.2$, $\Gamma / J_{\mathrm{D}}=0.22$, and $J / J_{\mathrm{D}}=0.01$ in $\hat{H}_{\mathrm{tSOC}}$ (Eq. 8). We plot the energy spectrum in Fig. 2(b) and once again find four modes pinned near the center of the bandgap at $E / J_{\mathrm{D}} \approx 1.0$. Like before, a plot of the real-space triplon density profile averaged over the four near-midgap states (Fig. 2(d)) shows that they reside at the corners and decay rapidly into the bulk, verifying that they are indeed corner modes. Also similar to the first model, we find edge modes with energies considerably away from the

\footnotetext{
3 In fact, the edge mode energies can be pushed into the bulk spectrum by tuning the parameters appropriately without destroying the corner modes.
} 
(a)

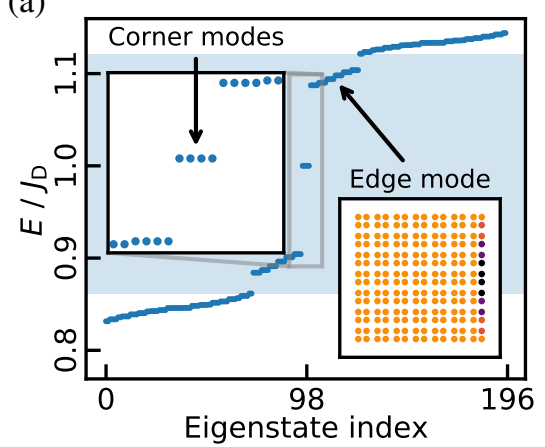

(b)

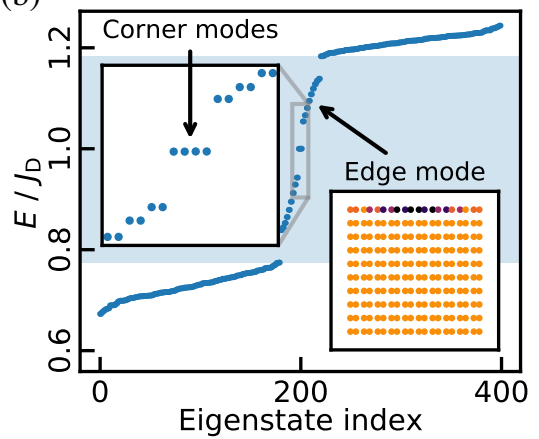

(c)

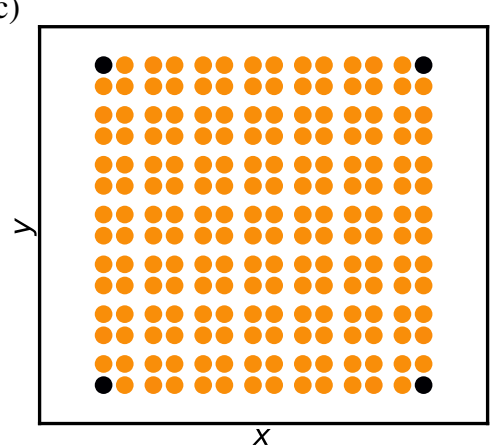

(d)

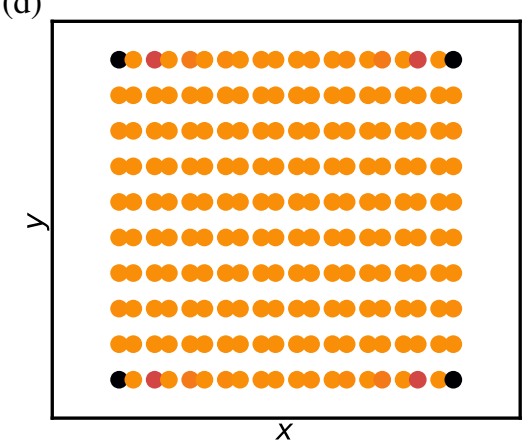

(e)

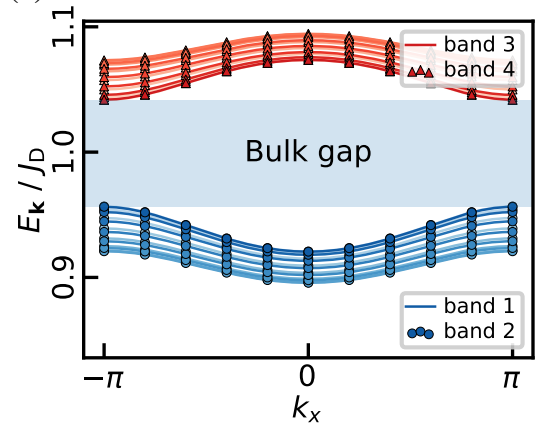

(f)

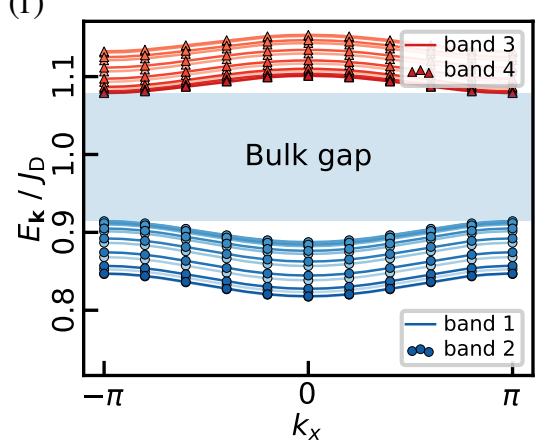

FIG. 2. Corner modes and Band dispersions: (a) Energy spectrum for the tBBH model with open boundary conditions (OBC), showing four corner modes (top inset) and edge modes inside the bulk bandgap (shaded region). The corner modes are pinned to the near-midgap energy $E / J_{\mathrm{D}} \approx 1$. The triplon density for a typical edge mode is given in the bottom-right inset. (b) Energy spectrum for the tSOC model in the HOT phase, with OBC, showing corner modes and edge modes like in (a). Figures (c) and (d) give the average triplon density profiles over all four corner modes of the tBBH and tSOC models, respectively. The triplon excitations are localized at the corners of the lattice (see Fig. 1(b), (d)). (e) and (f) show the band dispersions (orthographically projected onto the $k_{y}=0$ plane), and the bulk bandgap, for the triplon modes in the tBBH and tSOC models, respectively. The parameter values used for plotting the dispersions in (e) and (f) are $J_{1}^{x} / J_{\mathrm{D}}=J_{1}^{y} / J_{\mathrm{D}}=0.04, J_{2}^{x} / J_{\mathrm{D}}=J_{2}^{y} / J_{\mathrm{D}}=0.1$ and $J_{1} / J_{\mathrm{D}}=0.06, J_{2} / J_{\mathrm{D}}=0.1, J / J_{\mathrm{D}}=0.03, \Gamma / J_{\mathrm{D}}=0.11, D / J_{\mathrm{D}}=0.1$, respectively. The triplon dispersion for both the models comprises four Bloch bands, out of which bands 1 (lines) and 2 (०) form a degenerate pair and bands 3 (lines) and $4(\Delta)$ form another.

middle of (but inside) the bandgap for the second model. The density profile for a typical edge mode is given in Fig. 2(b) bottom inset.

Band structures: The band structures for the two models (Eqs. 7 and 9) are shown in Fig. 2(e) and (f), with respective parameter values discussed in the caption. In each model, the particle-like bosonic modes are made up of four bands (see the last paragraph of Sec. III). In the first model, the lowest two energy bands, i.e., bands $(1,2)$, are degenerate, and so are bands $(3,4)$; see Fig. 2(e). The bulk bandgap occurs between band pair $(1,2)$ and band pair $(3,4)$. The corner modes (see Fig. 2(a)) appear at an energy $E$ near the middle of this bandgap with $E \approx J_{\mathrm{D}}$, where the intra-dimer Heisenberg exchange $J_{\mathrm{D}}$ is the dominant energy scale. The bands for the second model are plotted in Fig. 2(f), and have the same type of degeneracies as the first model. Other features are also qualitatively similar to the first model, e.g., the bulk bandgap appearing between bands 2 and 3. Further details and explicit expressions for the band dispersions are discussed in app. A.

\section{A. Phase diagrams}

Having established the presence of corner modes for a set of parameter values, we now explore the full phase diagram for the two models. In particular, we look for ways to drive transitions from the HOT triplon phase to other phases with trivial (or possibly first-order) topology.

\section{Model I: $t B B H$ model}

In the fermionic $\mathrm{BBH}$ model, the complete phase diagram for the topological phases is accessed by allowing the hopping amplitudes on the $2 \mathrm{D}$ square lattice to be anisotropic; in other words, the amplitudes along the $x$ direction are tuned independently from those along the $y$ direction. Since our first model closely resembles the fermionic $\mathrm{BBH}$ model, we introduce a similar anisotropy into the tBBH model by making the Heisenberg exchange interactions along the horizontal ( $x$-direction) bonds independent from those along vertical ( $y$-direction) ones; see Fig. 1(b). This entails that the triplon Hamiltonian 
in Eq. 7 is redefined as

$$
\begin{aligned}
H_{\mathbf{k}}^{\mathrm{tBBH}} \stackrel{\text { redef. }}{=} & J_{\mathrm{D}} \sigma_{0} \eta_{0} \tau_{0}+\left(\frac{J_{1}^{x}}{2} \sigma_{1} \eta_{0}-\frac{J_{1}^{y}}{2} \sigma_{2} \eta_{2}\right)\left(\tau_{0}-\tau_{1}\right) \\
+ & \frac{J_{2}^{x}}{2}\left(\cos k_{x} \sigma_{1} \eta_{0}-\sin k_{x} \sigma_{2} \eta_{3}\right)\left(\tau_{0}-\tau_{1}\right) \\
& \quad-\frac{J_{2}^{y}}{2} \sigma_{2}\left(\cos k_{y} \eta_{2}+\sin k_{y} \eta_{1}\right)\left(\tau_{0}-\tau_{1}\right),
\end{aligned}
$$

where $J_{1,2}^{x}$ and $J_{1,2}^{y}$ represent the strengths of interactions on the $x$ and $y$ bonds, respectively. Continuing with the analogy between the tBBH and fermionic BBH models, we expect to drive a transition from the HOT phase to other phases by tuning the ratios $J_{1}^{x} / J_{2}^{x}$ and $J_{1}^{y} / J_{2}^{y}$. Setting $J_{2}^{x} / J_{\mathrm{D}}=J_{2}^{y} / J_{\mathrm{D}}=0.2$ in $H_{\mathbf{k}}^{\mathrm{tBBH}}$ (Eq. 10), we construct the phase diagram in Fig. 3(a) by separately tuning $J_{1}^{x} / J_{\mathrm{D}}$ and $J_{1}^{y} / J_{\mathrm{D}}$ from 0.0 to 0.4 . The HOT triplon phase occurs in the region with both $J_{1}^{x}<J_{2}^{x}$ and $J_{1}^{y}<J_{2}^{y}$. At any point in this region of parameter space, the system hosts stable corner modes. If either $J_{1}^{x} / J_{2}^{x}$ or $J_{1}^{y} / J_{2}^{y}$ are made greater than 1.0, the HOT phase is destroyed and the corner modes disappear. Notably, the phase boundary of the transition from a HOT to a nonHOT phase, indicated by the dashed lines $J_{1}^{x, y}=J_{2}^{x, y}$ in Fig. 3(a), does not coincide with a closing of the bulk bandgap! This unique feature of HOT phases underlines that they are truly distinct from conventional first-order topological phases. In the phase diagram of Fig. 3(a), the bulk bandgap (Fig. 2(e)), given by the formula

$$
\begin{aligned}
\Delta_{\text {bulk }} & =E_{+}^{(\min )}-E_{-}^{(\max )} \\
& =\frac{2 J_{\mathrm{D}} \sqrt{\left(\left|J_{1}^{x}\right|-\left|J_{2}^{x}\right|\right)^{2}+\left(\left|J_{1}^{y}\right|-\left|J_{2}^{y}\right|\right)^{2}}}{E_{+}^{(\min )}+E_{-}^{(\max )}},
\end{aligned}
$$

closes only at a single point when $J_{1}^{x} / J_{2}^{x}=J_{1}^{y} / J_{2}^{y}=1$ (see Fig. 3(a)). In Eq. 11, $E_{+}^{(\min )}$ and $E_{-}^{(\max )}$ (where $E_{+}^{(\min )} \geq E_{-}^{(\max )}>0$ ) are the maximum and minimum values for the bands 1,2 and 3,4 , respectively - explicit expressions are provided in app. A. Though the HOT phase boundaries are not marked by a closing of the bulk bandgap, they do coincide with bandgap closings in the edge theories of the model - this is further discussed in Sec. VI together with the topological invariants for our models.

\section{Model II: $t S O C$ model}

Moving on to constructing a phase diagram for our second model, the tuning parameters at our disposal to drive a transition are $J_{1}, J_{2}, D$ and $\Gamma$. We set $J$, the Heisenberg exchange on vertical bonds, to zero for the present discussion. Since the interactions along the $x$ direction are the same as in the first model, we once again choose the ratio $J_{1} / J_{2}$ as one of the parameters. Interestingly, tuning the strengths of $D$ or $\Gamma$ (as long as
$\left.D, \Gamma \ll J_{\mathrm{D}}\right)$ does not destroy the HOT-triplon phase! We have checked that the corner modes persist as long as both $D, \Gamma$ are not equal to zero. Therefore, we must introduce additional terms in the Hamiltonian (Eq. 3) to drive a transition. We explore two such avenues: (a) by applying a staggered Zeeman field

$$
\hat{H}_{\mathrm{stag}-B}^{\mathrm{spin}}=-h_{\mathrm{s}} \sum_{\mathbf{R}, a=1,2, l}(-1)^{a} S_{\mathbf{R}, a, l}^{z}
$$

of strength $h_{\mathrm{s}}$, or (b) by applying a homogeneous Zeeman field

$$
\hat{H}_{\mathrm{hom}-B}^{\mathrm{spin}}=-h \sum_{\mathbf{R}, a=1,2, l} S_{\mathbf{R}, a, l}^{z}
$$

controlled by the strength $h$. In the staggered field configuration, the Zeeman field at dimers 1 and 2 of the unit cell respectively points in the $-\hat{z}$ and $+\hat{z}$ directions, while for the homogeneous configuration it points everywhere in the $+\hat{z}$ direction (see Fig. 1(d)). The corresponding spin Hamiltonian in each case takes the form $\hat{H}_{\mathrm{tSOC}}^{\mathrm{spin}}+\hat{H}_{\mathrm{stag}-B}$ and $\hat{H}_{\mathrm{tSOC}}^{\mathrm{spin}}+\hat{H}_{\mathrm{hom}-B}$, respectively; the triplon Hamiltonians are discussed in app. A. We discuss the phase diagrams for the staggered and homogeneous field configurations separately.

a. Staggered field We begin with the staggered field, since the associated phase diagram will turn out to be analogous to that of the tBBH model. To investigate the phase diagram, we set $D / J_{\mathrm{D}}=\Gamma / J_{\mathrm{D}}=0.1$ and tune $h_{\mathrm{s}} / J_{\mathrm{D}}$ (the staggered-field strength) from 0.0 to 0.2 . Like for the first model, we tune $J_{1} / J_{\mathrm{D}}$ from 0.0 to 0.4 with $J_{2} / J_{\mathrm{D}}=0.2$. We show the resulting phases in Fig. 3(b). Indeed, we find that the HOT phase transitions to a different topological state when $h_{\mathrm{s}}$ becomes greater than a critical value $h_{\mathrm{s}}^{(\mathrm{c})} \approx D$. Also, like in the first model, the HOT-phase disappears when $J_{1}>J_{2}$. Both these transitions are in general not marked by a bulk bandgap (Fig. 2(f)) closing except at the point $J_{1}=J_{2}$ and

$$
h_{\mathrm{s}}=h_{\mathrm{s}}^{(\mathrm{c})}:=\frac{\sqrt{J_{\mathrm{D}}^{2}+2 D J_{\mathrm{D}}}-\sqrt{J_{\mathrm{D}}^{2}-2 D J_{\mathrm{D}}}}{2} ;
$$

see Fig. 3(b); see also app. A for the derivation of Eq. 14.

$b$. Homogeneous field Keeping all other parameters unchanged, we set $h_{\mathrm{s}}=0$ and tune $h / J_{\mathrm{D}}$ from 0.0 to 2.0 . The resulting phase diagram, shown in Fig. 3(c), is more complex than those encountered so far. There are now two critical field strengths $h_{\mathrm{c} 1}$ and $h_{\mathrm{c} 2}$, given by

$$
\begin{aligned}
& h_{\mathrm{c} 1}\left(J_{1}\right)=\left(\sqrt{J_{\mathrm{D}}^{2}+J_{\mathrm{D}} f_{-}\left(J_{1}\right)}-\sqrt{J_{\mathrm{D}}^{2}-J_{\mathrm{D}} f_{-}\left(J_{1}\right)}\right) / 2 \\
& h_{\mathrm{c} 2}\left(J_{1}\right)=\left(\sqrt{J_{\mathrm{D}}^{2}+J_{\mathrm{D}} f_{+}\left(J_{1}\right)}-\sqrt{J_{\mathrm{D}}^{2}-J_{\mathrm{D}} f_{+}\left(J_{1}\right)}\right) / 2 \\
& f_{-}\left(J_{1}\right)=\sqrt{4 D^{2}+\left(\left|J_{1}\right|-\left|J_{2}\right|\right)^{2}} \\
& f_{+}\left(J_{1}\right)=\sqrt{4 D^{2}+\left(\left|J_{1}\right|+\left|J_{2}\right|\right)^{2}},
\end{aligned}
$$



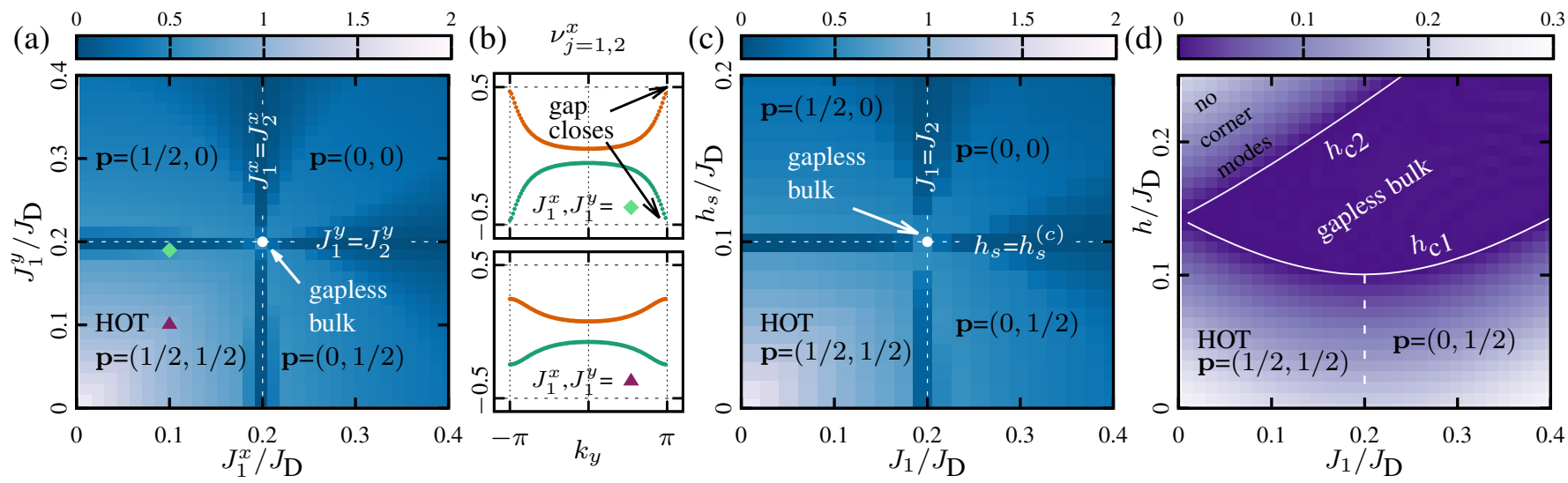

FIG. 3. Phase diagrams: (a) The phases of the BBH model for triplons (tBBH); the HOT-triplon phase appears when $J_{1}^{x, y}<J_{2}^{x, y}$. The Wannier bandgap (see Sec. VI), shown with a color-density plot, closes along the dashed lines $J_{1}^{x}=J_{2}^{x}, J_{1}^{y}=J_{2}^{y}$. The bulk bandgap closes only at the intersection point of the lines. The Wannier-sector polarizations $\mathbf{p}=\left(p_{x}, p_{y}\right)$ are quantized to either 0 or $1 / 2$ (see Sec. VI). (b) Gapless (top inset) and gapped (bottom inset) Wannier bands $\nu_{j}^{x}(\mathbf{k})$ appearing in phases close to the horizontal-transition line $(\diamond)$ and inside the HOT region $(\triangle)$ in panel (a), respectively. (c) Phases of the second model with SOC triplons (tSOC) in a staggered field $h_{\mathrm{s}}$. The phases are in one-to-one correspondence with the tBBH model in panel (a); the staggered-field strength $h_{\mathrm{s}}$ acts like $J_{1}^{y}$ from panel (a). (d) Phase diagram for the tSOC model in a homogeneous field $h$; a HOT phase is found when $h<h_{\mathrm{c} 1}$ and $J_{1}<J_{2}$. The Wannier-sector polarizations $\mathbf{p}$ are quantized for $h<h_{\mathrm{c} 1}$. The bulk bandgap (shown with a color-density plot) closes when $h_{\mathrm{c} 1} \leq h \leq h_{\mathrm{c} 2}$, and the system cannot harbor protected near-midgap modes. Beyond $h>h_{\mathrm{c} 2}$, the system is gapped but does not have any corner modes. In panels (c) and (d), the parameter values $D / J_{\mathrm{D}}=\Gamma / J_{\mathrm{D}}=0.1$ are used.

that separate the various phases; see app. A for derivations. The HOT triplon phase persists as long as $h<h_{\mathrm{c} 1}$ and $J_{1}<J_{2}$. Similar to the previous cases, the system transitions from a HOT phase to one with no corner modes when $J_{1}$ is tuned past $J_{2}$, with the bulk bandgap remaining open in the process. However, for $h_{\mathrm{c} 1}<h<h_{\mathrm{c} 2}$, the bulk bandgap is absent, thereby precluding the existence of protected near-midgap states of any order (edge states or corner states). Beyond $h>h_{\mathrm{c} 2}$, the bulk bandgap reopens but the HOT phase is not recovered for any values of $J_{1}$ and $J_{2}$.

The phases encountered in the above phase diagrams, especially those without robust corner modes, can be further characterized using topological invariants. We develop this characterization in more detail in Sec. VI on topological invariants.

\section{SYMMETRIES}

Usually, a set of non-commuting symmetries protect the HOT phases hosting corner modes [1]. For example, in the two-dimensional fermionic BBH model, there exists a pair of mirror-like symmetries $\mathcal{M}_{x}$ (reflection about the $y$ axis) and $\mathcal{M}_{y}$ (reflection about the $x$ axis) that anti-commute [1]. As we shall demonstrate below, like in the BBH model, usual (commuting) mirror symmetries preclude the existence of an HOT phase in the spindimer models we consider, whereas non-commuting mirror symmetries allow for HOT. We now discuss analogues of these non-commuting mirror-like symmetries for our two models below.

\section{A. Model I: tBBH model}

For the first model, we find that the canonical mirror operation $M_{x}$, i.e., reflection about a line parallel to the $y$ axis and passing through the center of a unit cell (Fig. 1(b)), is not a symmetry by itself. Therefore, following the mirror reflection, we perform the operation $\tilde{C}_{2}^{z}$, a $\pi$ spin rotation about the $z$ axis acting only on the spins of dimers 1 and 3 (Fig. 1(b)), making them transform as

$$
\left[\begin{array}{l}
S_{\mathbf{R},(1,3), \ell}^{x} \\
S_{\mathbf{R},(1,3), \ell}^{y} \\
S_{\mathbf{R},(1,3), \ell}^{z}
\end{array}\right] \stackrel{M_{x}}{\rightarrow}\left[\begin{array}{r}
S_{\mathbf{R}^{\prime},(3,1), \ell}^{x} \\
-S_{\mathbf{R}^{\prime},(3,1), \ell}^{y} \\
-S_{\mathbf{R}^{\prime},(3,1), \ell}^{z}
\end{array}\right] \stackrel{\tilde{C}_{\rightrightarrows}^{z}}{\rightarrow}\left[\begin{array}{r}
-S_{\mathbf{R}^{\prime},(3,1), \ell}^{x} \\
S_{\mathbf{R}^{\prime},(3,1), \ell}^{y} \\
-S_{\mathbf{R}^{\prime},(3,1), \ell}^{z}
\end{array}\right],
$$

while the spins in dimers 2 and 4 transform as

$$
\left[\begin{array}{l}
S_{\mathbf{R},(2,4), \ell}^{x} \\
S_{\mathbf{R},(2,4), \ell}^{y} \\
S_{\mathbf{R},(2,4), \ell}^{z}
\end{array}\right] \stackrel{M_{x}}{\rightarrow}\left[\begin{array}{r}
S_{\mathbf{R}^{\prime},(4,2), \ell}^{x} \\
-S_{\mathbf{R}^{\prime},(4,2), \ell}^{y^{2}} \\
-S_{\mathbf{R}^{\prime},(4,2), \ell}^{z}
\end{array}\right]
$$

where $\mathbf{R}=i_{x} \hat{x}+i_{y} \hat{y}$ and $\mathbf{R}^{\prime}=M_{x}(\mathbf{R})=-i_{x} \hat{x}+i_{y} \hat{y}$ is the mirror image of $\mathbf{R}$ under $M_{x}$. The layer index $\ell$ takes the values $T$ or $B$ for spins in the top or bottom layers, respectively. In app. C, we show that the composite transformation $\tilde{C}_{2}^{z} \circ M_{x}$ is a symmetry of the Hamiltonian. As an example, we focus on the transformation of a term with ferromagnetic interactions in Eq. 3, such as

$$
H_{\mathbf{R}}^{(x, y)}:=J_{1} S_{\mathbf{R}, 1, \ell}^{(x, y)} S_{\mathbf{R}, 4, \ell}^{(x, y)}-J_{1} S_{\mathbf{R}, 2, \ell}^{(x, y)} S_{\mathbf{R}, 3, \ell}^{(x, y)}
$$


which transforms as

$$
\begin{aligned}
H_{\mathbf{R}}^{(x, y)} & \stackrel{M_{x}}{\rightarrow} J_{1} S_{\mathbf{R}^{\prime}, 3, \ell}^{(x, y)} S_{\mathbf{R}^{\prime}, 2, \ell}^{(x, y)}-J_{1} S_{\mathbf{R}^{\prime}, 4, \ell}^{(x, y)} S_{\mathbf{R}^{\prime}, 1, \ell}^{(x, y)} \\
& \stackrel{\tilde{C}_{2}^{z}}{\rightarrow}-J_{1} S_{\mathbf{R}^{\prime}, 3, \ell}^{(x, y)} S_{\mathbf{R}^{\prime}, 2, \ell}^{(x, y)}+J_{1} S_{\mathbf{R}^{\prime}, 4, \ell}^{(x, y)} S_{\mathbf{R}^{\prime}, 1, \ell}^{(x, y)} \\
& =H_{\mathbf{R}^{\prime}}^{(x, y)} .
\end{aligned}
$$

We see from above, since $H_{\mathbf{R}}^{(x, y)} \stackrel{\tilde{C}_{2}^{z} \circ M_{x}}{\rightarrow} H_{\mathbf{R}^{\prime}}^{(x, y)}$, where $\mathbf{R}^{\prime}$ is a primitive lattice vector, the sum $\sum_{\mathbf{R}} H_{\mathbf{R}}^{(x, y)}$ remains invariant under the transformation. Similarly, the sum of the rest of the Heisenberg interaction terms in Eq. 2 also remains invariant, see app. C for further details. This establishes $\tilde{C}_{2}^{z} \circ M_{x}$ as a one of the mirror-like symmetries for our first model.

Searching for the second symmetry, we find that the usual-mirror reflection $M_{y}$, about a line parallel to the $x$ axis and bisecting a unit cell, leaves the spin Hamiltonian in Eq. 2 invariant. Under this symmetry operation, the spins transform as

$$
\begin{aligned}
& {\left[\begin{array}{l}
S_{\mathbf{R},(2,3), \ell}^{x} \\
S_{\mathbf{R},(2,3), \ell}^{y} \\
S_{\mathbf{R},(2,3), \ell}^{z}
\end{array}\right] \stackrel{M_{y}}{\rightarrow}\left[\begin{array}{r}
-S_{\mathbf{R}^{\prime},(3,2), \ell}^{x} \\
S_{\mathbf{R}^{\prime},(3,2), \ell} \\
-S_{\mathbf{R}^{\prime},(3,2), \ell}^{z}
\end{array}\right]} \\
& {\left[\begin{array}{l}
S_{\mathbf{R},(1,4), \ell}^{x} \\
S_{\mathbf{R},(1,4), \ell}^{y} \\
S_{\mathbf{R},(1,4), \ell}^{z}
\end{array}\right] \stackrel{M_{y}}{\rightarrow}\left[\begin{array}{r}
-S_{\mathbf{R}^{\prime},(4,1), \ell}^{x} \\
S_{\mathbf{R}^{\prime},(4,1), \ell}^{y} \\
-S_{\mathbf{R}^{\prime},(4,1), \ell}^{z}
\end{array}\right],}
\end{aligned}
$$

where $\mathbf{R}^{\prime}=M_{y}(\mathbf{R})=i_{x} \hat{x}-i_{y} \hat{y}$. We provide further details on the invariance of the terms in Eq. 2 under $M_{y}$ in app. C.

The two symmetries $\tilde{C}_{2}^{z} \circ M_{x}$ and $M_{y}$ that we found above do not mix the triplon flavors, but admit different representations in the three decoupled triplon flavor sectors. As previously stated, we shall focus on the $t_{x}$ and $t_{y}$ sectors since these are the sectors that contribute to the HOT triplon phase. In the $t_{x}$ sector, the matrix representations $\mathcal{M}_{x}^{(x)}$ and $\mathcal{M}_{y}^{(x)}$ for the symmetries that transform the bosonic Nambu vector $\Phi_{\mathbf{R}, x}$ in Eq. 6 are

$$
\begin{aligned}
\tilde{C}_{2}^{z} \circ M_{x} & \equiv \mathcal{M}_{x}^{(x)}=-\sigma_{1} \eta_{3} \tau_{0} \\
M_{y} & \equiv \mathcal{M}_{y}^{(x)}=-\sigma_{1} \eta_{1} \tau_{0},
\end{aligned}
$$

whereas for the $t_{y}$ sector, the matrix representations $\mathcal{M}_{x}^{(y)}$ and $\mathcal{M}_{y}^{(y)}$ operate as

$$
\begin{aligned}
\tilde{C}_{2}^{z} \circ M_{x} & \equiv \mathcal{M}_{x}^{(y)}=\sigma_{1} \eta_{3} \tau_{0} \\
M_{y} & \equiv \mathcal{M}_{y}^{(y)}=\sigma_{1} \eta_{1} \tau_{0}
\end{aligned}
$$

on $\Phi_{\mathbf{R}, y}$. In both cases, the Bloch Hamiltonians for Model I in Eqs. 7 and 10 respectively satisfy $H_{\mathbf{k}}^{\mathrm{tBBH}}=$ $\mathcal{M}_{\alpha}^{(\beta)}{ }^{\dagger} H_{M_{\alpha} \mathbf{k}}^{\mathrm{tBBH}} \mathcal{M}_{\alpha}^{(\beta)}$, where $\alpha, \beta \in\{x, y\}$, leaving the full Hamiltonian invariant. Using the representations for the symmetry operations in Eq. 20, we find $\left\{\mathcal{M}_{x}^{(\alpha)}, \mathcal{M}_{y}^{(\alpha)}\right\}=$ 0 , where $\alpha \in\{x, y\}$, making the non-commuting property of the symmetries manifest, and hence fulfilling one of the main requirements for obtaining a HOT phase.

\section{B. Model II: tSOC model}

To discuss the symmetries of our second model, we define the DM and $\Gamma$ interactions terms from Eq. 3 at the lattice position $(\mathbf{R}, a)$ as

$$
\begin{aligned}
D_{\mathbf{R}, a} & =\sum_{l}(-1)^{a} D\left(S_{\mathbf{R}, a, \ell}^{y} S_{\mathbf{R}+\hat{y}, a, \ell}^{x}-S_{\mathbf{R}, a, \ell}^{x} S_{\mathbf{R}+\hat{y}, a, \ell}^{y}\right), \\
\Gamma_{\mathbf{R}, a} & =\sum_{l}(-1)^{a} \Gamma\left(S_{\mathbf{R}, a, \ell}^{y} S_{\mathbf{R}+\hat{y}, a, \ell}^{x}+S_{\mathbf{R}, a, \ell}^{x} S_{\mathbf{R}+\hat{y}, a, \ell}^{y}\right),
\end{aligned}
$$

respectively. A mirror reflection $\left(M_{x}\right)$ about a line parallel to the $y$ axis and passing through the center of a unit cell transforms the spin operators as

$$
\left[\begin{array}{l}
S_{\mathbf{R},(1,2), \ell}^{x} \\
S_{\mathbf{R},(1,2), \ell}^{y} \\
S_{\mathbf{R},(1,2), \ell}^{z}
\end{array}\right] \stackrel{M_{x}}{\longrightarrow}\left[\begin{array}{r}
S_{\mathbf{R}^{\prime},(2,1), \ell}^{x} \\
-S_{\mathbf{R}^{\prime},(2,1), \ell}^{y} \\
-S_{\mathbf{R}^{\prime},(2,1), \ell}^{z}
\end{array}\right]
$$

where $\mathbf{R}^{\prime}=M_{x}(\mathbf{R})$, or more explicitly $\mathbf{R}=i_{x} \hat{x}+i_{y} \hat{y}$ and $\mathbf{R}^{\prime}=-i_{x} \hat{x}+i_{y} \hat{y}$. Therefore, under $M_{x}$, the DM terms $D_{\mathbf{R}, a}$ transforms according to $D_{\mathbf{R}, 1} \stackrel{M_{x}}{\rightarrow} D_{\mathbf{R}^{\prime}, 2}$ and $D_{\mathbf{R}, 2} \stackrel{M_{x}}{\rightarrow} D_{\mathbf{R}^{\prime}, 1}$ - that is, up to a primitive lattice translation, the DM term for dimer 1 maps to the term for dimer 2, and vice-versa. Since $\mathbf{R}^{\prime}$ is a primitive lattice vector, the sum $\sum_{\mathbf{R}, a} D_{\mathbf{R}, a}$ over lattice vectors remains invariant; the same goes for the sum of Gamma interactions $\sum_{\mathbf{R}, a} \Gamma_{\mathbf{R}, a}$. The other terms in Eq. 3 can be shown to remain invariant as well (see app. C for details), implying that $M_{x}$ is indeed a symmetry of the Hamiltonian in Eq. 3.

The second symmetry for the model is constructed by composing two operations: a mirror reflection $M_{y}$ about the $x$ axis passing between two unit cells followed by a $\pi / 2$ global spin rotation $C_{4}^{z}$ about the $\hat{z}$ direction. The spin operators transform as

$$
\left[\begin{array}{c}
S_{\mathbf{R}, a, \ell}^{x} \\
S_{\mathbf{R}, a, \ell}^{y} \\
S_{\mathbf{R}, a, \ell}^{z}
\end{array}\right] \stackrel{M_{y}}{\rightarrow}\left[\begin{array}{r}
-S_{\mathbf{R}^{\prime}, a, \ell}^{x} \\
S_{\mathbf{R}^{\prime}, a, \ell}^{y} \\
-S_{\mathbf{R}^{\prime}, a, \ell}^{z}
\end{array}\right] \stackrel{C_{4}^{z}}{\rightarrow}\left[\begin{array}{r}
S_{\mathbf{R}^{\prime}, a, \ell}^{y} \\
S_{\mathbf{R}^{\prime}, a, \ell}^{x} \\
-S_{\mathbf{R}^{\prime}, a, \ell}^{z}
\end{array}\right],
$$

where, $\mathbf{R}^{\prime}=M_{y}(\mathbf{R})=i_{x} \hat{x}-i_{y} \hat{y}$. As a result, the DM and $\Gamma$ terms transform according to $D_{\mathbf{R}_{1}, a} \rightarrow D_{\mathbf{R}_{2}, a}$ and $\Gamma_{\mathbf{R}_{1}, a} \rightarrow \Gamma_{\mathbf{R}_{2}, a}$, where $\mathbf{R}_{1}=i_{x} \hat{x}+i_{y} \hat{y}$ and $\mathbf{R}_{2}=$ $i_{x} \hat{x}-\left(i_{y}+1\right) \hat{y}$. Since $\mathbf{R}_{2}$ is a primitive lattice vector, the sums $\sum_{\mathbf{R}, a} D_{\mathbf{R}, a}$ and $\sum_{\mathbf{R}, a} \Gamma_{\mathbf{R}, a}$ are invariant under $C_{4}^{z} \circ M_{y}$. Similarly, the Heisenberg terms in Eq. 3 can be shown to remain invariant as well (see app. C), thereby establishing $C_{4}^{z} \circ M_{y}$ as another mirror-like symmetry for our second model.

The non-commuting nature of the two symmetries $M_{x}$ and $C_{4}^{z} \circ M_{y}$ can be verified by writing their representations in the subspace of triplons contributing to the HOT phase, i.e. the $x$ and $y$ triplons. In this subspace, apart from changing $\mathbf{R} \rightarrow M_{\alpha}(\mathbf{R})$, the symmetries are 
represented by matrices

$$
\begin{aligned}
M_{x} & \equiv \mathcal{M}_{x}=\sigma_{1} \eta_{3} \tau_{0} \\
C_{4}^{z} \circ M_{y} & \equiv \mathcal{M}_{y}=\sigma_{0} \eta_{1} \tau_{0},
\end{aligned}
$$

which act on the column vector $\Psi_{\mathbf{R}}$ (see Eq. 8), and constrain the Bloch Hamiltonian $H_{\mathbf{k}}^{\mathrm{tSOC}}$ as $H_{\mathbf{k}}^{\mathrm{tSOC}}=$ $\mathcal{M}_{\alpha}^{\dagger} H_{M_{\alpha} \mathbf{k}}^{\mathrm{tSOC}} \mathcal{M}_{\alpha}$, where $\alpha \in\{x, y\}$. The matrices $\mathcal{M}_{x}$ and $\mathcal{M}_{y}$ satisfy $\left\{\mathcal{M}_{x}, \mathcal{M}_{y}\right\}=0$ and hence, as expected, do not commute.

In the presence of a Zeeman field, one or both of the symmetries break depending on whether a staggered field $h_{\mathrm{s}}$ (Eq. 12) or homogeneous field $h$ (Eq. 13) is applied. For the staggered field case, $M_{x}$ (or $\mathcal{M}_{x}$ ) is preserved, while $C_{4}^{z} \circ M_{y}\left(\mathcal{M}_{y}\right)$ is broken. The latter gets replaced by $\mathcal{T} \circ C_{4}^{z} \circ M_{y}$, i.e., $C_{4}^{z} \circ M_{y}$ followed by a time-reversal operation $\mathcal{T}$, as the new symmetry. When a homogeneous field is applied, the new symmetry $\mathcal{T} \circ C_{4}^{z} \circ M_{y}$ also works as the replacement for the broken $C_{4}^{z} \circ M_{y}$ symmetry. However this time, in addition to $C_{4}^{z} \circ M_{y}$, the symmetry $M_{x}$ also breaks and is replaced by $\mathcal{T} \circ M_{x}$. The matrix representations for the new symmetries in the relevant triplon sectors are obtained by left-multiplying the complex-conjugation operator $K$ with the matrices $\mathcal{M}_{x}, \mathcal{M}_{y}$ in Eq. 24. The modified representations still continue to anti-commute, see app. C for details.

\section{TOPOLOGICAL INVARIANTS}

It has been shown $[1,51]$ that we can characterize the $1 \mathrm{D}$ edge of a $2 \mathrm{D}$ fermionic HOT phase using standard firstorder topological invariants such as polarization [52]. In turn, the bulk of the HOT phase can then be characterized using the invariants of their effective edge theories. The idea of using edge polarization as a topological invariant has been generalized to bosonic systems in the context of magnons [22, 25]. Here, we apply this framework to the triplon systems introduced in Sec. III; however, we remark here that the term polarization is used to refer to the topological invariant, and not to a physical polarization of the system. It has been demonstrated $[1$, 51] that the topological nature of the edge polarizations can be characterized using Wannier Hamiltonians (a bulk property) without introducing physical edges in the model. We can obtain the Wannier Hamiltonians by constructing Wilson loop operators $\mathcal{W}_{\mathbf{k}}^{\alpha}$ that traverse the first Brillouin zone (BZ) once along either the $\alpha=x$ or $y$ directions starting from a base momentum $\mathbf{k}$. Defining the overlap matrix

$$
F_{\alpha \mathbf{k}}=\left[F_{\alpha \mathbf{k}}\right]_{m, n}:=\left\langle u_{m, \mathbf{k}+\Delta \mathbf{k}}|\Sigma| u_{n, \mathbf{k}}\right\rangle
$$

for each momentum $\mathbf{k}$, the loop operator $\mathcal{W}_{\mathbf{k}}^{\alpha}$ is conveniently expressed as an ordered matrix product of the overlap matrices

$$
\mathcal{W}_{\mathbf{k}}^{\alpha}=F_{\alpha, \mathbf{k}+N_{\alpha} \Delta \mathbf{k}} \cdots F_{\alpha, \mathbf{k}+\Delta \mathbf{k}} F_{\alpha \mathbf{k}}
$$

following the loop. Here, $\Sigma:=1_{n} \otimes \tau_{3}$ is the symplectic identity, $\left|u_{n, \mathbf{k}}\right\rangle$ are the eight-component particle-like Bloch vectors obtained by solving the Bloch Hamiltonians of Eq. 9 and 10, $N_{\alpha=x, y}$ are the number of unit cells along the $x$ or $y$ directions and $\Delta \mathbf{k}=2 \pi \hat{\alpha} / N_{\alpha}$. The band indices $m$ and $n$ label the rows and columns of the matrix $F_{\alpha \mathbf{k}}$ and run over a subset of bands in the system which we choose to be bands 1 and 2 for each our models (see Fig. 2(e), (f)). This choice of bands is guided by the analogy with the fermionic BBH model, for which bands 1 and 2 would be the filled bands. We mention here that, unlike in the fermion version, $F_{\alpha \mathbf{k}}$ is defined in terms of an inner product with matrix $\Sigma^{4}$; a similar definition has been used in the context of magnons - another bosonic BdG system - in Ref. 22.

The Wannier Hamiltonians $H_{\mathcal{W}^{\alpha}}(\mathbf{k})$, one for each direction $x$ and $y$, are defined by the loop operators via

$$
\exp \left(2 \pi \mathrm{i} H_{\mathcal{W}^{\alpha}}(\mathbf{k})\right):=\mathcal{W}_{\mathbf{k}}^{\alpha} .
$$

The Wannier Hamiltonian for the direction $\alpha$ is adiabatically connected (topologically equivalent) to the physical Hamiltonian for the edge perpendicular to $\alpha[1,53]$, allowing us to use it to characterize the edge theories. The eigenvalues of $H_{\mathcal{W}^{\alpha}}(\mathbf{k})$ give the Wannier bands $\nu_{j}^{\alpha}(\mathbf{k})$ $(j=1,2)$, which are defined modulo 1 , whereas the eigenvectors $\left|\nu_{j}^{\alpha}(\mathbf{k})\right\rangle$ can be used to obtain $[1,53]$ the Wannier band subspace spanned by the states $\left|w_{j}^{\alpha}(\mathbf{k})\right\rangle$ in the original eight-component Nambu basis . Here, we have used the same notation for denoting Wannier bands, subspaces, etc. as in Ref. 1. The bands $\nu_{j}^{\alpha}(\mathbf{k})$, constructed from Wilson loops along the direction $\alpha$, depend only on the momentum component perpendicular to $\alpha$; e.g., $\nu_{j}^{x}\left(k_{x}, k_{y}\right)$ depends on $k_{y}$ only. Since the matrices $F_{\alpha \mathbf{k}}$ in each of our models are constructed using Bloch bands 1 and 2, the number of Wannier bands at any given $\mathbf{k}$ is two.

A useful feature of the Wannier bands is that they are gapped inside a HOT phase and become gapless when the underlying system transitions to a different topological phase. The gap closing of the Wannier bands is not necessarily accompanied with the closing of the bulk bandgap. As a result, the associated states $\left|w_{j}^{\alpha}(\mathbf{k})\right\rangle$ can carry topological inavriants of their own. For the class of 2D HOT phases that we consider in this paper, this topological invariant was shown to be the polarization of the Wannier bands, or Wannier-sector polarization [1]. To elaborate on this further, we focus on the Wannier Hamiltonian $H_{\mathcal{W}^{x}}$ for the Wilson loops along the $x$ direction. If we define the overlap amplitude $F^{\mathrm{pol}}{ }_{j, \mathbf{k}}=\left\langle w_{j}^{\alpha}(\mathbf{k}+\Delta \mathbf{k})|\Sigma| w_{j}^{\alpha}(\mathbf{k})\right\rangle$ (where $\Delta \mathbf{k}=\left(0,2 \pi / N_{y}\right)$ ), we can construct yet another set of Wilson loops,

$$
\tilde{\mathcal{W}}_{y, k_{x}}^{j}=F_{j, \mathbf{k}+N_{y} \Delta \mathbf{k}}^{\mathrm{pol}} \cdots F_{j, \mathbf{k}}^{\mathrm{pol}},
$$

\footnotetext{
4 The paraunitarity condition implies that the (particle-like) eigenstates at a given $\mathbf{k}$ are orthonormal with respect to this inner product with metric $\Sigma$.
} 
one for each Wannier band $j \in\{1,2\}$, that run along the $y$ direction. This time, however, the loops are formulated using the Wannier band basis $\left|w_{j}^{\alpha}(\mathbf{k})\right\rangle$ instead of the Bloch states $\left|u_{n, \mathbf{k}}\right\rangle$ (see Eq. 25) and the overlap amplitudes $F_{j, \mathbf{k}}^{\mathrm{pol}}$ are scalars instead of matrices. Following the convention in Ref. 1, we call these new loops nested Wilson loops. Since the $F^{\mathrm{pol}}{ }_{j, \mathbf{k}}$ are scalars, the product $\tilde{\mathcal{W}}_{y, k_{x}}^{j}$ only depends on $k_{x}$. The polarization for the states $\left|w_{j}^{x}(\mathbf{k})\right\rangle$ can be written in terms of the nested Wilson loops as follows:

$$
p_{y}^{\left(\nu_{j}^{x}\right)}=-\frac{\mathrm{i}}{2 \pi N_{x}} \sum_{k_{x}} \log \tilde{\mathcal{W}}_{y, k_{x}}^{j}
$$

The Wannier-sector polarization $p_{y}^{\left(\nu_{j}^{x}\right)}$, defined modulo 1 , characterizes the topology on the $x$ edge. A value of $p_{y}^{\left(\nu_{j}^{x}\right)}=0$ indicates that the edge has a trivial topology, while $p_{y}^{\left(\nu_{j}^{x}\right)}=1 / 2$ implies a non-trivial edge topology. Similar to the fermionic $\mathrm{BBH}$ model, the quantization of $p_{y}^{\left(\nu_{j}^{x}\right)}$ is a result of the constraints imposed on the polarization due to the mirror-like symmetries we introduced in Sec. V. The symmetries also constrain the polarizations of the two Wannier bands, $j \in\{1,2\}$, to be equal, i.e., $p_{y}^{\left(\nu_{1}^{x}\right)}=p_{y}^{\left(\nu_{2}^{x}\right)}$ $\bmod 1$. Therefore, we refer to the Wannier-sector polarizations $p_{y}^{\left(\nu_{1,2}^{x}\right)}$ collectively as $p_{y}$. We have verified numerically that these constraints hold. In contrast to the fermionic case, our definition of the Wannier-sector polarization, given in Eq. 28, uses the symplectic identity $\Sigma$ instead of the usual matrix identity. Similar definitions for computing polarizations of Bloch bands [54] and of Wannier bands [25] in bosonic BdG systems can be found in existing literature.

Following the same set of steps, we can define the Wannier-sector polarization $p_{x}$ starting from the Wannier Hamiltonian $H_{\mathcal{W}^{y}}(\mathbf{k})$ to characterize the $y$-edge topology. The polarization $p_{x}$ will also satisfy the same symmetry constraints as $p_{y}$, and will take values 0 or $1 / 2$ modulo 1 . Therefore, we can use the pair of Wannier-sector polarizations $\mathbf{p}=\left(p_{x}, p_{y}\right)$ to further classify the HOT triplon phases that appear in the phase-diagrams (Fig. 3) of our models.

a. Model I ( $\mathrm{BBBH}$ model): Numerically computing the Wannier-sector polarizations for the first model, we find the HOT-triplon phase in Fig. 3(a), obtained when $J_{1}^{x}<J_{2}^{x}$ and $J_{1}^{y}<J_{2}^{y}$, has $\mathbf{p}=(1 / 2,1 / 2)$, implying that both the $y$-edge and $x$-edge polarizations are quantized to $1 / 2$. In the HOT phase, the Wannier bands $\nu_{j=1,2}^{\alpha}(\mathbf{k})$ for both the $\alpha \in\{x, y\}$ directions are gapped. We show the two gapped bands $\nu_{j}^{x}(\mathbf{k})$, calculated from Wilson loops running along the $x$ direction, in Fig. 3(b) bottom inset. Transitions to other phases occur when either the $x$ - or $y$-direction Wannier-band gap closes modulo 1 . For example, when $J_{1}^{y} \rightarrow J_{2}^{y}$, the gap between the bands $\nu_{j}^{x}(\mathbf{k}), j \in\{1,2\}$, begins to close, as seen from Fig. 3(b) top inset. Hence, the line $J_{1}^{y}=J_{2}^{y}$ is a phase boundary (see dashed horizontal line in Fig. 3(a)). Similarly, the line $J_{1}^{x}=J_{2}^{x}$ (represented by a dashed vertical line) forms another phase boundary, along which the gap between the Wannier bands $\nu_{j}^{y}(\mathbf{k})(j=1,2)$ closes. The Wanniersector polarizations of the resulting additional phases are $\mathbf{p}=(0,1 / 2)$ when $J_{1}^{y}>J_{2}^{y}$ and $J_{1}^{x}<J_{2}^{x}, \mathbf{p}=$ $(1 / 2,0)$ when $J_{1}^{y}<J_{2}^{y}$ and $J_{1}^{x}>J_{2}^{x}$, and $\mathbf{p}=(0,0)$ when both $J_{1}^{x, y}>J_{2}^{x, y}$. Therefore, the phases of Fig. 3(a) are classified as $\mathbb{Z}_{2} \times \mathbb{Z}_{2}$, exactly like the fermion BBH model. The dependence of the minimum of the two Wannier-band gaps, $\min \left\{\left(\nu_{j=1}^{x}-\nu_{j=2}^{x}\right) \bmod 1,\left(\nu_{j=1}^{y}-\nu_{j=2}^{y}\right) \bmod 1\right\}$, on $J_{1}^{x}, J_{1}^{y}$ is shown as a density plot in the same figure.

b. Model II (tSOC model): For our second model, where the HOT phase of SOC triplons is destroyed by applying either a staggered or a homogeneous Zeeman field, we find two different topological classifications. The Wannier-sector polarizations $\mathbf{p}$ for the phases in Fig. 3(c), obtained when a staggered Zeeman field $h_{\mathrm{s}}$ is applied, are in one-to-one correspondence with the phase diagram (Fig. 3(a)) for our first model. The only difference is that the role of $J_{1}^{y}$ in the first model is now fulfilled by the staggered field strength $h_{\mathrm{s}}$, and $J_{1,2}^{x}$ are relabelled as $J_{1,2}$, respectively; the line $h_{\mathrm{s}}=h_{\mathrm{s}}^{(\mathrm{c})}$ (Eq. 14) now marking one of the phase boundaries (see dashed-horizontal line in Fig. 3(c)). Therefore, the topological phases for the staggered-field case are again classified as $\mathbb{Z}_{2} \times \mathbb{Z}_{2}$. Moving on to the phase diagram for the homogeneous field in Fig. 3(d), we find that we can classify the phases using the Wannier-sector polarization $\mathbf{p}$ only when $h<h_{\mathrm{c} 1}$. The HOT phase that occurs when $J_{1}<J_{2}$ and $h<h_{\mathrm{c} 1}$ has polarizations $\mathbf{p}=(1 / 2,1 / 2)$. Like the previous cases, the transition to a phase with $\mathbf{p}=(0,1 / 2)$ takes place via a Wannier-band gap closing along the vertical line segment $J_{1}=J_{2}$ (dashed line Fig. $3(\mathrm{~d})$ ). When $h_{\mathrm{c} 1} \leq h \leq h_{\mathrm{c} 2}$, the bulk is gapless and therefore the system cannot host in-gap states. Beyond $h>h_{\mathrm{c} 2}$, although the bulk becomes gapped once again, it is hard to determine from numerics whether the Wannier bands are truly gapped. Consequently, the numerical calculation of the Wannier sector polarizations also becomes challenging. Nonetheless, in this region of parameter space, real-space numerics do not reveal any protected edge states or corner states. This may indicate that the construction of the Wannier Hamiltonian using only two Bloch bands 1, 2 may no longer be adequate and must be generalized to include all four bands 1 to 4 to explore this region of the phase diagram; this will be explored in future work.

Before we end this section, we mention that the Wannier-sector polarizations can be calculated without involving the $\Sigma$ matrix if we work in the limit of large dimer strength $J_{\mathrm{D}}$. In this limit, cross amplitudes between the particle-like and hole-like sectors in the BdG Hamiltonians (Eqs. 10, 9) get suppressed as $\mathcal{O}\left(1 / J_{\mathrm{D}}\right)$ and we can work with the Hamiltonian projected on to the particle sector (such a projection is discussed in Sec. VIIB and app. B 2 a). The projected Hamiltonian is Hermitian and can be diagonalized using unitary matrices instead of paraunitary ones. Since the non-trivial topology arises in the excited triplon sector of our models, the projected 
Hamiltonian, for each model, is topologically equivalent and adiabatically connected to the original unprojected Hamiltonian. Hence, both the Hamiltonians share the same topological invariants for the triplon sector. The eigenstates of the projected Hamiltonian are orthonormal with respect to the standard inner product, and therefore the original fermionic definitions for Wilson loops, etc., can be used to calculate the Wannier-sector polarizations. We have numerically verified that this gives the same answer as the previous analysis.

\section{EDGE THEORY AND SHARED CORNER MODES}

The corner modes of a HOT phase show additional features compared to edge modes at the junction of a 1D trivial and a first-order topological system. In particular, the HOT-corner modes are shared by the topological edge theories that intersect at the corner. This shared nature of a corner mode can be verified by finding the effective continuum theories of the intersecting edges, e.g., right $(x=0)$ and top $(y=0)$ edges (see Fig. 1(b), (d)), and then introducing corners into the resulting 1D theories. Hence, the corner can be considered as the edge of a 1D edge theory. Since the edge theory of a $2 \mathrm{D}$ HOT phase is described by first-order topology, the corner mode can be recovered following a Jackiw-Rebbi-type [1, 55] approach and tuning a mass-like term across zero at some point along the spatial direction. We now describe finding a shared corner mode for each of our two models in more detail.

\section{A. Model I: tBBH model}

We work with the anisotropic version of the model again (see Eq. 10) in which the couplings $J_{1,2}^{x}$ along the $\hat{x}$ direction are in general distinct from the couplings $J_{1,2}^{y}$ along $\hat{y}$. Doing so allows us to isolate the edge Hamiltonian for the $x=0$ edge from the $y=0$ edge and vice-versa. We obtain the continuum Hamiltonian,

$$
\begin{aligned}
\tau_{3} H_{\mathrm{cont}}^{\mathrm{tBBH}}= & J_{\mathrm{D}} \sigma_{0} \eta_{0} \tau_{3}+\left(m_{x} \sigma_{1} \eta_{0}+v_{x}\left(-\mathrm{i} \partial_{x}\right) \sigma_{2} \eta_{3}\right. \\
& \left.-m_{y} \sigma_{2} \eta_{2}+v_{y}\left(-\mathrm{i} \partial_{y}\right) \sigma_{2} \eta_{1}\right)\left(\tau_{3}-\mathrm{i} \tau_{2}\right)
\end{aligned}
$$

by expanding the Bloch Hamiltonian in Eq. 10 around the bandgap-closing momentum $\mathbf{k}_{0}$, which for positive $J_{1,2}^{x, y}$ is $\mathbf{k}_{0}=(\pi, \pi)$ (see app. A), and then taking the continuum limit by transforming $q_{x} \rightarrow-\mathrm{i} \partial_{x}$ and $q_{y} \rightarrow-\mathrm{i} \partial_{y}$, where $\mathbf{q}=\left(q_{x}, q_{y}\right)$ is a small deviation from $\mathbf{k}_{0}$. The mass-like terms $m_{x}$ and $m_{y}$ in Eq. 30 are related to the original couplings as $m_{x}=\left(J_{1}^{x}-J_{2}^{x}\right) / 2$ and $m_{y}=\left(J_{1}^{y}-J_{2}^{y}\right) / 2$, while the coefficients $v_{x}$ and $v_{y}$ are given by $v_{x}=J_{2}^{x} / 2$, $v_{y}=J_{2}^{y} / 2$. Assuming positive $v_{x}$ and $v_{y}$, when the mass term $m_{x}\left(m_{y}\right)<0$, the phase on the $y=0(x=0)$ edge is topological, while $m_{x}\left(m_{y}\right)>0$ makes the phase trivial.
Setting $m_{x}\left(m_{y}\right)=0$ produces a gapless phase on the $y=0$ $(x=0)$ edge. Therefore, we obtain the Hamiltonian of the $y=0$ (top) edge,

$$
\tau_{3} H_{\mathrm{top}}^{\mathrm{tBBH}}=J_{\mathrm{D}} \sigma_{0} \tau_{3}+\left(m_{x} \sigma_{1}+v_{x}\left(-\mathrm{i} \partial_{x}\right) \sigma_{2}\right)\left(\tau_{3}-\mathrm{i} \tau_{2}\right),
$$

by introducing a domain wall $m_{y}(x, y)=\tanh (y)$ along the top edge, and solving for modes that disperse along the $x$ direction but decay exponentially in the $y$ direction, see app. B for details. The exact function chosen to model domain walls like $m_{y}(x, y)$ is not critical to our analysis; any function $f(y)$ interpolating between $m_{y}<0$ (topological) and $m_{y}>0$ (trivial) with a single zero crossing is a valid choice. The corner mode for $\tau_{3} H_{\text {top }}^{\mathrm{tBBH}}$ can now be obtained by inserting yet another domain wall $m_{x}(x)=\tanh (x)$ and looking for a single trapped mode. This process (see app. B) gives

$$
\psi_{\text {top-right }}=\left[\begin{array}{l}
1 \\
0
\end{array}\right]^{(\sigma)} \otimes\left[\begin{array}{l}
1 \\
0
\end{array}\right]^{(\eta)} \otimes\left[\begin{array}{l}
1 \\
0
\end{array}\right]^{(\tau)}
$$

as the corner mode living on the top-right corner that has the near-mid-bandgap energy $E=J_{\mathrm{D}}$. To find the corner mode supported by the right $(x=0)$ edge, we reverse the order in which we introduce the domain walls. To be more specific, we first get the continuum Hamiltonian $\tau_{3} H_{\text {right }}^{\mathrm{tBBH}}$ for the right edge by inserting the domain wall $m_{x}(x, y)=$ $\tanh (x)$, this time running along the right edge. We then introduce the second domain wall $m_{y}(y)=\tanh (y)$ in the $1 \mathrm{D}$ Hamiltonian $\tau_{3} H_{\text {right }}^{\mathrm{tBBH}}$ and find, yet again, a single trapped mode $\psi_{\text {right-top }}$ at the near-mid-bandgap energy $E=J_{\mathrm{D}}$, see app. B for the explicit calculation. Comparing $\psi_{\text {top-right }}$ with $\psi_{\text {right-top }}$ we find that they are identical, thus confirming that the corner mode is shared between the top and right edges.

\section{B. Model II: tSOC model}

We now turn to the corner mode in the tSOC model: we wish to show that it too is shared between the edge theories. As suggested by the similarity between the phase diagram of the tSOC model in the presence of a staggered field (Fig. 3(b)) and that of the tBBH model (Fig. 3(a)), the staggered-field strength $h_{\mathrm{s}}$ can serve as a tuning parameter for introducing edges into the tSOC model-specifically, $h_{\mathrm{s}}$ plays a role analogous to that of $J_{1}^{y}$ in the tBBH model. With this in mind, we develop an analysis similar to the one in Sec. VII A, starting from the Bloch Hamiltonian

$$
H_{h_{\mathrm{s}}}^{\mathrm{tSOC}}(\mathbf{k})=H_{\mathbf{k}}^{\mathrm{tSOC}}-h_{\mathrm{s}} \sigma_{3} \eta_{2} \tau_{3}
$$

where $H_{\mathbf{k}}^{\mathrm{tSOC}}$ was defined in Eq. 9. The band structure for $H_{h_{\mathrm{s}}}^{\mathrm{tSOC}}(\mathbf{k})$ in the HOT phase typically looks like Fig. 2(f) even in the presence of a staggered field, i.e., bands 1 and 2 are degenerate and separated by a bulk bandgap (for 
$D, \Gamma \neq 0$ ) from bands 3 and 4 which are also degenerate. The bulk bandgap closes at the gap-closing momentum $\mathbf{k}_{0}=(\pi, \pi / 2)$ when $J_{1}=J_{2}$ and $h_{\mathrm{s}}=h_{\mathrm{s}}^{(\mathrm{c})}$ (see Eq. 14 as well as app. A), and critical staggered field $h_{\mathrm{s}}^{(\mathrm{c})} \approx D$ when $|D| \ll J_{\mathrm{D}}$. The continuum Hamiltonian in the neighborhood of $\mathbf{k}_{0}$ is

$$
\begin{aligned}
\tau_{3} H_{\mathrm{cont}}^{\mathrm{tSOC}} & =J_{\mathrm{D}} \sigma_{0} \eta_{0} \tau_{3} \\
+ & \left(\frac{J_{1}-J_{2}}{2} \sigma_{1}-\frac{J_{2}}{2}\left(-\mathrm{i} \partial_{x}\right) \sigma_{2}\right) \eta_{0}\left(\tau_{3}-\mathrm{i} \tau_{2}\right) \\
& -\sigma_{3} \eta_{2}\left(h_{\mathrm{s}} \tau_{0}-D\left(\tau_{3}-\mathrm{i} \tau_{2}\right)\right) \\
& -\Gamma\left(-\mathrm{i} \partial_{y}\right) \sigma_{3} \eta_{1}\left(\tau_{3}-\mathrm{i} \tau_{2}\right),
\end{aligned}
$$

where we have assumed $J=0$ for simplicity. We have checked that the outcome of the calculation does not depend on $J$ provided $|J|<|\Gamma|$; see app. B for details.

Identifying mass terms in Eq. 34 analogous to the ones in Eq. 30 is straightforward if we work in the large- $J_{\mathrm{D}}$ limit. This is justified because the dominant energy scale $J_{\mathrm{D}}$ sets the size of the gap between the singlet ground state and triplon excitations, meaning the model is asymptotically connected to the $J_{\mathrm{D}} \rightarrow \infty$ limit. Consequently, the finite- $J_{\mathrm{D}}$ model is topologically equivalent to the leading-order Hamiltonian in the large- $J_{\mathrm{D}}$ limit. The latter Hamiltonian is obtained by projecting the finite$J_{\mathrm{D}}$ Hamiltonian onto the particle-like sector of the BdG space - in effect performing a first-order Schrieffer-Wolff transformation (Refs. 56-58, also see app. B) onto the positive eigenstates of $J_{\mathrm{D}} \sigma_{0} \eta_{0} \tau_{3}$. The effective (Hermitian) Hamiltonian describing the positive-energy bands is then

$$
\begin{aligned}
\tilde{H}_{\mathrm{cont}}^{\mathrm{tSOC}}=J_{\mathrm{D}} \sigma_{0} \eta_{0}+ & \left(m_{x} \sigma_{1}-v_{x}\left(-\mathrm{i} \partial_{x}\right) \sigma_{2}\right) \eta_{0} \\
& -\sigma_{3}\left(m_{y} \eta_{2}+v_{y}\left(-\mathrm{i} \partial_{y}\right) \eta_{1}\right)
\end{aligned}
$$

where $m_{x}=\left(J_{1}-J_{2}\right) / 2, m_{y}=h_{\mathrm{s}}-D, v_{x}=J_{2} / 2$, and $v_{y}=\Gamma$. Since the critical field $h_{\mathrm{s}}^{(\mathrm{c})}$ approaches $D$ in the large- $J_{\mathrm{D}}$ limit, the mass term $m_{y}$ indeed marks the topological transition that for finite $J_{\mathrm{D}}$ happens at $h_{\mathrm{s}}=h_{\mathrm{s}}^{(\mathrm{c})}$, represented by the horizontal dashed line in Fig. 3(c).

We find the top-edge Hamiltonian $\tilde{H}_{\text {top }}^{\mathrm{tSOC}}$ following the same steps as for the tBBH model: we first introduce a domain wall $m_{y}(x, y)=\tanh y$ along the top edge at $y=0$, and then write down the effective Hamiltonian

$$
\tilde{H}_{\mathrm{top}}^{\mathrm{tSOC}}=J_{\mathrm{D}} \sigma_{0}+m_{x} \sigma_{1}-v_{x}\left(-\mathrm{i} \partial_{x}\right) \sigma_{2}
$$

for states that are exponentially confined to this top edge. As we did for the tBBH model, we get to the corner mode for the current model by introducing another domain wall $m_{x}(x)=\tanh x$, leading to the mode

$$
\psi_{\text {top-right }}=\left[\begin{array}{l}
0 \\
1
\end{array}\right]^{(\sigma)} \otimes\left[\begin{array}{l}
0 \\
1
\end{array}\right]^{(\eta)} \otimes\left[\begin{array}{l}
1 \\
0
\end{array}\right]^{(\tau)}
$$

localized at the top-right corner and having the midgap energy $E=J_{\mathrm{D}}$.

Proceeding in reverse order, i.e., starting by introducing a right edge in the form of $m_{x}(x, y)=\tanh x$, leads to the $1 \mathrm{D}$ edge Hamiltonian $\tilde{H}_{\text {right }}^{\mathrm{tSOC}}$. An edge (or corner) is then introduced in this edge Hamiltonian by setting $m_{y}(y)=$ tanh $y$, leading to the corner mode $\psi_{\text {right-top }}=\psi_{\text {top-right }}$. Once again, this shows the corner mode is shared between the top and right edge theories.

\section{SUMMARY AND OUTLOOK}

We have shown in this paper that dimerized quantum paramagnets can host triplon modes which exhibit higher order topology and corner states at mid-bandgap energies. The $2 \mathrm{D}$ models proposed and studied here are spin variants of previously explored fermionic Hamiltonians or obtained by a staggered stacking of 1D dimer Hamiltonians which have been shown to exhibit first-order topology. We have provided numerical support for corner modes and the phase diagram of such HOT triplon phases and their transitions. In addition, we have provided analytical results on their symmetry protection and edge theories, and constructed their topological invariants. Our theoretical work thus opens up a new direction in the study of dimerized quantum magnets.

The wealth of quantum dimer magnets which have been explored over the years [26-36] makes it is very likely that model Hamiltonians of the type we have studied can be realized in experiments. Identifying specific materials candidates is a natural next step worth investigating. A possible guiding principle to narrow down the search for suitable candidate systems would be to look for materials with noncommuting mirror symmetries like those discussed in Sec. V, rather than usual mirror symmetries.

Another important open question, not substantially addressed in previous research on HOT phases in magnetic systems, is one of detecting the near-midgap energy corner modes. One possible route to probing such localized nonzero-energy spin excitations might be to excite them selectively via terahertz radiation [59, 60], which can access typical magnetic exchange energy scales, and look for mid-bandgap energy terahertz absorption that scales with the number of corners in the sample. Another approach would be to look at site resolved spin-spin correlations using spin-Hall noise spectroscopy [61]. Further, it might be possible to employ recent advances in atomically resolved electron spin resonance measurements to probe the spatial localization of the excitations [62], or recently developed scanning techniques [63-66] that can use nitrogen-vacancy centers in diamond to measure local magnetic moments and extract dynamical information via spin noise. In this context, the rare earth dimer systems [36] could prove useful owing to the reduced exchange energy scales in these systems, which makes them amenable to such experimental techniques. 


\section{ACKNOWLEDGMENTS}

We acknowledge funding from the Natural Sciences and Engineering Research Council (NSERC) of Canada. We gratefully recognize the computational resources provided to us by Compute Canada; in particular on the Niagara, Graham, and Cedar systems. G. M. acknowledges funding from le Fonds de recherche du Québec - Nature et technologies.

\section{Appendix A: Band structures}

In this section, we elaborate on the main features of the triplon band dispersions for our two models that we refer to in the main text.

\section{Model I: tBBH model}

The (doubly degenerate) eigenvalues of $\tau_{3} H_{\mathbf{k}}^{\mathrm{tBBH}}$ (from Eq. 10) are $-E_{\mathbf{k},+},-E_{\mathbf{k},+},-E_{\mathbf{k},-},-E_{\mathbf{k},-}, E_{\mathbf{k},-}, E_{\mathbf{k},-}$, $E_{\mathbf{k},+}$, and $E_{\mathbf{k},+}$, where

$$
\begin{aligned}
E_{\mathbf{k}, \pm}^{2}=J_{\mathrm{D}}^{2} \pm & J_{\mathrm{D}}\left(J_{1}^{x^{2}}+2 J_{1}^{x} J_{2}^{x} \cos k_{x}+J_{2}^{x^{2}}\right. \\
& \left.+J_{1}^{y 2}+2 J_{1}^{y} J_{2}^{y} \cos k_{y}+J_{2}^{y^{2}}\right)^{1 / 2} .
\end{aligned}
$$

The minimum of $E_{\mathbf{k},+}$ and maximum of $E_{\mathbf{k},-}$ are respectively

$$
\begin{aligned}
& E_{+}^{(\min )}=\sqrt{J_{\mathrm{D}}^{2}+J_{\mathrm{D}} \sqrt{\left(\left|J_{1}^{x}\right|-\left|J_{2}^{x}\right|\right)^{2}+\left(\left|J_{1}^{y}\right|-\left|J_{2}^{y}\right|\right)^{2}}} \\
& E_{-}^{(\max )}=\sqrt{J_{\mathrm{D}}^{2}-J_{\mathrm{D}} \sqrt{\left(\left|J_{1}^{x}\right|-\left|J_{2}^{x}\right|\right)^{2}+\left(\left|J_{1}^{y}\right|-\left|J_{2}^{y}\right|\right)^{2}}},
\end{aligned}
$$

showing that the bulk bandgap between $E_{-}$and $E_{+}$given in Eq. 11 closes iff $\left|J_{1}^{x}\right|=\left|J_{2}^{x}\right|$ and $\left|J_{1}^{y}\right|=\left|J_{2}^{y}\right|$. Furthermore, the closing occurs at momentum $\mathbf{k}_{0}=\left(k_{0 x}, k_{0 y}\right)$, where

$$
\begin{aligned}
& k_{0 x}=\left\{\begin{array}{ll}
\pi & \text { if } J_{1}^{x}=J_{2}^{x} \\
0 & \text { if } J_{1}^{x}=-J_{2}^{x}
\end{array},\right. \\
& k_{0 y}=\left\{\begin{array}{ll}
\pi & \text { if } J_{1}^{y}=J_{2}^{y} \\
0 & \text { if } J_{1}^{y}=-J_{2}^{y}
\end{array} .\right.
\end{aligned}
$$

\section{Model II: tSOC model}

In the absence of a Zeeman field, the (doubly degenerate) eigenvalues of $\tau_{3} H_{\mathbf{k}}^{\mathrm{tSOC}}$ are $-E_{\mathbf{k},+},-E_{\mathbf{k},+},-E_{\mathbf{k},-}$,

$$
\begin{aligned}
& -E_{\mathbf{k},-}, E_{\mathbf{k},-}, E_{\mathbf{k},-}, E_{\mathbf{k},+}, \text { and } E_{\mathbf{k},+}, \text { where } \\
& E_{\mathbf{k}, \pm}^{2}=J_{\mathrm{D}}^{2}+2 J_{\mathrm{D}} J \cos k_{y} \\
& \pm J_{\mathrm{D}}\left(2 D^{2}\left(1-\cos \left(2 k_{y}\right)\right)+2 \Gamma^{2}\left(1+\cos \left(2 k_{y}\right)\right)\right. \\
& \left.\quad+J_{1}^{2}+2 J_{1} J_{2} \cos k_{y}+J_{2}^{2}\right)^{1 / 2}
\end{aligned}
$$

There exists a full bandgap betwen $E_{\mathbf{k},-}$ and $E_{\mathbf{k},+}$ unless $\left|J_{1}\right|=\left|J_{2}\right|$ and at least one of $D$ and $\Gamma$ is zero. In our work, we assume $D$ and $\Gamma$ are both nonzero, keeping the bands gapped at zero field even if $J_{1}=J_{2}$. Furthermore, nonzero $D$ and $\Gamma$ is necessary to realize the HOT phase in this model. This is consistent with the findings in Ref. 43, albeit in the context of first-order topology.

In the presence of a staggered Zeeman field (see Eq. 12), the triplon Bloch Hamiltonian is given by

$$
H_{h_{\mathrm{s}}}^{\mathrm{tSOC}}(\mathbf{k})=H_{\mathbf{k}}^{\mathrm{tSOC}}-h_{\mathrm{s}} \sigma_{3} \eta_{2} \tau_{3},
$$

while for a uniform field configuration (see Eq. 13), we have

$$
H_{h}^{\mathrm{tSOC}}(\mathbf{k})=H_{\mathbf{k}}^{\mathrm{tSOC}}-h \sigma_{0} \eta_{2} \tau_{3},
$$

where $H_{\mathbf{k}}^{\mathrm{tSOC}}$ was defined in Eq. 9. The Hamiltonian $H_{h_{\mathrm{s}}}^{\mathrm{tSOC}}(\mathbf{k})$ for the staggered field was already reported in Eq. 33.

For both field configurations, the model does not admit a closed-form dispersion relation except at certain momenta. We now discuss the details of these band dispersions and critical field strengths in the presence of staggered and homogeneous Zeeman fields individually.

\section{a. Staggered Zeeman field}

In the presence of a staggered Zeeman field $h_{\mathrm{s}}$, numerical investigations show that the eight bands of $\tau_{3} H_{h_{\mathrm{s}}}^{\mathrm{tSOC}}(\mathbf{k})$ (see Eq. 33) remain doubly degenerate. The investigations also show that band touchings occur iff $\left|J_{1}\right|=$ $\left|J_{2}\right|$ and $\left|h_{\mathrm{s}}\right|=\left|h_{\mathrm{s}}^{(\mathrm{c})}\right|$, where $h_{\mathrm{s}}^{(\mathrm{c})}:=\left(\sqrt{J_{\mathrm{D}}^{2}+2 J_{\mathrm{D}} D}-\right.$ $\left.\sqrt{J_{\mathrm{D}}^{2}-2 J_{\mathrm{D}} D}\right) / 2 \approx D$ for $|D| \ll J_{\mathrm{D}}$. The band touchings occur at $\mathbf{k}_{0}=\left(k_{0 x}, k_{0 y}\right)$, where

$$
\begin{aligned}
& k_{0 x}= \begin{cases}\pi & \text { if } J_{1}=J_{2} \\
0 & \text { if } J_{1}=-J_{2}\end{cases} \\
& k_{0 y}=\left\{\begin{array}{rl}
\frac{\pi}{2} & \text { if } h_{\mathrm{s}}=h_{\mathrm{s}}^{(\mathrm{c})} \\
-\frac{\pi}{2} & \text { if } h_{\mathrm{s}}=-h_{\mathrm{s}}^{(\mathrm{c})}
\end{array} .\right.
\end{aligned}
$$

Analytical values for the crossings can be found from the closed-form expressions for the eigenvalues of $\tau_{3} H_{h_{\mathrm{s}}}^{\mathrm{tSOC}}(\mathbf{k})$ for $\left|J_{1}\right|=\left|J_{2}\right|$ at the above momenta. 


\section{b. Homogeneous Zeeman field}

In the presence of a homogeneous Zeeman field $h$, the twofold degeneracy of the bands is broken. Numerical diagonalization of Eq. A5b shows that band touchings occur only along the $k_{y}= \pm \pi / 2$ lines. In terms of $f\left(k_{x}\right):=\sqrt{4 D^{2}+J_{1}^{2}+2 J_{1} J_{2} \cos k_{x}+J_{2}^{2}}$, the (particlelike) eigenvalues along $k_{y}= \pm \pi / 2$ are

$$
\begin{aligned}
& E_{1}\left(k_{x}, \pm \pi / 2\right)=\sqrt{J_{\mathrm{D}}^{2}-J_{\mathrm{D}} f\left(k_{x}\right)}-h \\
& E_{2}\left(k_{x}, \pm \pi / 2\right)=\sqrt{J_{\mathrm{D}}^{2}-J_{\mathrm{D}} f\left(k_{x}\right)}+h \\
& E_{3}\left(k_{x}, \pm \pi / 2\right)=\sqrt{J_{\mathrm{D}}^{2}+J_{\mathrm{D}} f\left(k_{x}\right)}-h \\
& E_{4}\left(k_{x}, \pm \pi / 2\right)=\sqrt{J_{\mathrm{D}}^{2}+J_{\mathrm{D}} f\left(k_{x}\right)}+h .
\end{aligned}
$$

With the degeneracy lifted, the near-midgap states now occur within the bandgap between $E_{2}$ and $E_{3}$.

Note that the maximum and minimum values reached by $f\left(k_{x}\right)$ are respectively $f_{+}$and $f_{-}$, where

$$
\begin{aligned}
& f_{+}=\sqrt{4 D^{2}+\left(\left|J_{1}\right|+\left|J_{2}\right|\right)^{2}} \\
& f_{-}=\sqrt{4 D^{2}+\left(\left|J_{1}\right|-\left|J_{2}\right|\right)^{2}} .
\end{aligned}
$$

Using $f_{ \pm}$we define the two critical field values:

$$
\begin{aligned}
h_{\mathrm{c} 1} & :=\frac{\sqrt{J_{\mathrm{D}}^{2}+J_{\mathrm{D}} f_{-}}-\sqrt{J_{\mathrm{D}}^{2}-J_{\mathrm{D}} f_{-}}}{2}, \\
h_{\mathrm{c} 2} & :=\frac{\sqrt{J_{\mathrm{D}}^{2}+J_{\mathrm{D}} f_{+}}-\sqrt{J_{\mathrm{D}}^{2}-J_{\mathrm{D}} f_{+}}}{2} .
\end{aligned}
$$

It is now straightforward to see that there is a bandgap between $E_{2}$ and $E_{3}$ for $|h|<h_{\mathrm{c} 1}$ and again for $|h|>h_{\mathrm{c} 2}$. In the remaining regions (i.e. $h_{\mathrm{c} 1} \leq|h| \leq h_{\mathrm{c} 2}$ ), the middle bands touch with linear crossing points, except for the critical values themselves, at which the crossings are linear in one momentum direction and quadratic in the other.

\section{Appendix B: Derivation of effective edge Hamiltonians for triplons}

In this appendix, we provide more details on the derivation of the effective edge theories and shared corner modes presented in Sec. VII. For each model, the effective edge Hamiltonians are obtained from the continuum theories taken about their respective bulk-bandgap-closing momenta. We discuss these details for the two models individually.

\section{Model I: tBBH model}

\section{a. Effective edge theories}

Starting from the continuum Hamiltonian about $\mathbf{k}_{0}=$ $(\pi, \pi)$ (Eq. 30) we isolate the "top" edge by introducing

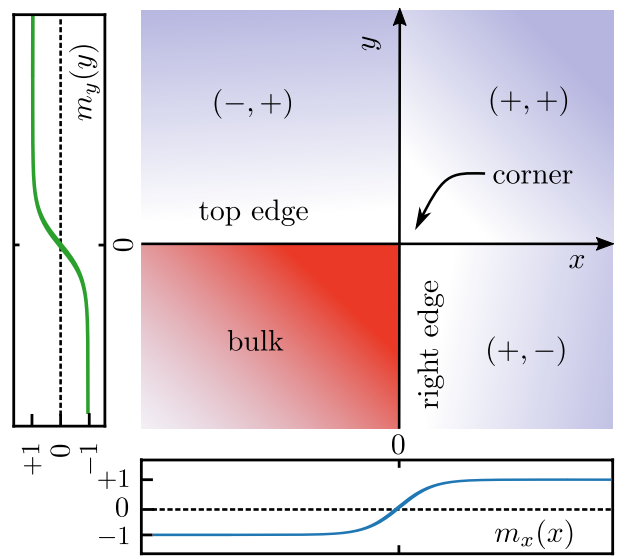

FIG. 4. Schematic representation of the mass terms $m_{x}, m_{y}$, as functions of position, that are used to isolate the corner mode shared by the top- and right-edge Hamiltonians.

a domain wall $m_{y}(y)$ (see Fig. 4) and seek edge states along the wall using a Jackiw-Rebbi ansatz,

$$
\psi_{q_{x}}(x, y)=\mathrm{e}^{-\gamma_{\text {top }} \int_{0}^{y} \mathrm{~d} \tilde{y} m_{y}(\tilde{y})} \mathrm{e}^{\mathrm{i} x q_{x}} \psi_{\text {top }}\left(q_{x}\right),
$$

where $q_{x}$ is the momentum along the edge and $\psi_{\text {top }}\left(q_{x}\right)$ is an eight-component vector independent of $m_{y}(y)$, to be determined below. In the main text, we chose $m_{y}(y)=\tanh y$ for simplicity; however, the choice of $m_{y}$ is not unique. In principle, any smooth function $m_{y}(y)$ interpolating between $m_{y}<0$ (topological) and $m_{y}>0$ (trivial) with a single zero crossing is an equally valid choice for a domain wall. Since $\psi_{q_{x}}(x, y)$ is meant to be an eigenvector of $\tau_{3} H_{\mathrm{cont}}^{\mathrm{tBBH}}$, it must satisfy

$$
\tau_{3} H_{\mathrm{cont}}^{\mathrm{tBBH}} \psi_{q_{x}}(x, y)=E_{q_{x}} \psi_{q_{x}}(x, y),
$$

where the eigenvalue $E_{q_{x}}$ gives the dispersion for the edge modes. If Eq. B2 is to have a non-trivial solution such that $\psi_{\text {top }}\left(q_{x}\right)$ is unique regardless of the choice of $m_{y}(y)$, $\psi_{\text {top }}\left(q_{x}\right)$ must be an element of $\operatorname{ker}\left(\sigma_{2}\left(\eta_{2}-\mathrm{i} \gamma_{\text {top }} v_{y} \eta_{1}\right)\left(\tau_{3}-\right.\right.$ $\left.\left.\mathrm{i} \tau_{2}\right)\right)$. Nontrivial (normalizable) solutions of this sort exist only for $\gamma_{\text {top }}=1 / v_{y}$ (see Eq. 30 for $v_{y}$ ), and are of the form

$$
\psi_{\mathrm{top}}\left(q_{x}\right)=\chi_{q_{x}}^{(\sigma)} \otimes\left[\begin{array}{l}
1 \\
0
\end{array}\right]^{(\eta)} \otimes \chi_{q_{x}}^{(\tau)}
$$

where $\chi_{q_{x}}^{(\sigma)}$ and $\chi_{q_{x}}^{(\tau)}$ are arbitrary two-component vectors. With this choice, the effective Hamiltonian for the top edge is

$$
\tau_{3} H_{\mathrm{top}}^{\mathrm{tBBH}}=J_{\mathrm{D}} \sigma_{0} \tau_{3}+\left(m_{x} \sigma_{1}+v_{x} q_{x} \sigma_{2}\right)\left(\tau_{3}-\mathrm{i} \tau_{2}\right),
$$

where $v_{x}$ was defined below Eq. 30. We reported the above Hamiltonian for the top edge in Eq. 31 of the main text.

Alternatively, we can introduce a "right" edge with a domain wall $m_{x}(x)$ (see Fig. 4). Using a Jackiw-Rebbi ansatz

$$
\psi_{q_{y}}(x, y)=\mathrm{e}^{-\gamma_{\text {right }} \int_{0}^{x} \mathrm{~d} \tilde{x} m_{x}(\tilde{x})} \mathrm{e}^{\mathrm{i} y q_{y}} \psi_{\text {right }}\left(q_{y}\right),
$$


we seek eigenvectors of $\tau_{3} H_{\mathrm{cont}}^{\mathrm{tBBH}}$ :

$$
\tau_{3} H_{\mathrm{cont}}^{\mathrm{tBBH}} \psi_{q_{y}}(x, y)=E_{q_{y}} \psi_{q_{y}}(x, y),
$$

where the eigenvalues $E_{q_{y}}$ give the dispersion for the topedge modes. Again, for the ansatz to yield a solution regardless of the details of $m_{x}(x), \psi_{\text {right }}\left(q_{y}\right)$ must be part of $\operatorname{ker}\left(\left(\sigma_{1} \eta_{0}+\mathrm{i} \gamma_{\text {right }} v_{x} \sigma_{2} \eta_{3}\right)\left(\tau_{3}-\mathrm{i} \tau_{2}\right)\right)$. Solutions arise only if $\gamma_{\text {right }}=1 / v_{x}$. Though the space of solutions is of the same dimension as in Eq. B3, $\psi_{\text {right }}\left(q_{y}\right)$ does not factor in the same way. Instead, solutions are of the form

$$
\psi_{\text {right }}\left(q_{y}\right)=\left(c_{1}\left(q_{y}\right) v_{1}+c_{2}\left(q_{y}\right) v_{2}\right) \otimes \chi_{q_{y}}^{(\tau)},
$$

where $c_{1}\left(q_{y}\right)$ and $c_{2}\left(q_{y}\right)$ are arbitrary $q_{y}$-dependent coefficients, $\chi_{q_{y}}^{(\tau)}$ is an arbitrary two-component vector and

$$
v_{1}:=\left[\begin{array}{l}
1 \\
0
\end{array}\right]^{(\sigma)} \otimes\left[\begin{array}{l}
1 \\
0
\end{array}\right]^{(\eta)}, \quad v_{2}:=\left[\begin{array}{l}
0 \\
1
\end{array}\right]^{(\sigma)} \otimes\left[\begin{array}{l}
0 \\
1
\end{array}\right]^{(\eta)} .
$$

The effective Hamiltonian for the right edge, now written in the basis of $\left(v_{1}, v_{2}\right)$ with Pauli matrices $\tilde{\sigma}_{\alpha}$, is

$$
\tau_{3} H_{\text {right }}^{\mathrm{tBBH}}=J_{\mathrm{D}} \tilde{\sigma}_{0} \tau_{3}+\left(m_{y} \tilde{\sigma}_{1}+v_{y} q_{y} \tilde{\sigma}_{2}\right)\left(\tau_{3}-\mathrm{i} \tau_{2}\right) .
$$

\section{b. $\quad$ Shared corner modes}

The corner mode for the top edge can now be obtained by inserting yet another domain wall in $m_{x}(x)$ at $x=0$ in the Hamiltonian $\tau_{3} H_{\text {top }}^{\mathrm{tBBH}}$ and looking for a single trapped mode. Specifically, we look for eigenstates of the form

$$
\begin{array}{r}
\psi_{\text {top }}(x)=\mathrm{e}^{-\gamma_{\text {top }- \text { right }} \int_{0}^{x} \mathrm{~d} \tilde{x} m_{x}(\tilde{x})} \psi_{\text {top-right }}, \\
\psi_{\text {top-right }}=\chi^{(\sigma)} \otimes\left[\begin{array}{l}
1 \\
0
\end{array}\right]^{(\eta)} \otimes \chi^{(\tau)} .
\end{array}
$$

For the solution to exist regardless of the details of $m_{x}(x)$, it should be part of $\operatorname{ker}\left(\sigma_{1}+\mathrm{i} \gamma_{\text {top-right }} v_{x} \sigma_{2}\right)\left(\tau_{3}-\mathrm{i} \tau_{2}\right)$. Furthermore, nontrivial solutions are of the form

$$
\psi_{\text {top-right }}=\left[\begin{array}{l}
1 \\
0
\end{array}\right]^{(\sigma)} \otimes\left[\begin{array}{l}
1 \\
0
\end{array}\right]^{(\eta)} \otimes \chi^{(\tau)}
$$

and exist only if $\gamma_{\text {top-right }}=1 / v_{x}$. Two such solutions are obtained, and are related by particle-hole conjugation. The positive-energy solution is

$$
\psi_{\text {top-right }}=\left[\begin{array}{l}
1 \\
0
\end{array}\right]^{(\sigma)} \otimes\left[\begin{array}{l}
1 \\
0
\end{array}\right]^{(\eta)} \otimes\left[\begin{array}{l}
1 \\
0
\end{array}\right]^{(\tau)}
$$

and appears at the near-midgap energy $E=J_{\mathrm{D}}$

On the other hand, the corner mode for the right edge is found by introducing a domain wall $m_{y}(y)$ in the Hamiltonian $\tau_{3} H_{\text {right }}^{\mathrm{tBBH}}$. We use the ansatz

$$
\begin{gathered}
\tilde{\psi}_{\text {right }}(y)=\mathrm{e}^{-\gamma_{\text {right-top }} \int_{0}^{y} \mathrm{~d} \tilde{y} m_{y}(\tilde{y})} \tilde{\psi}_{\text {right-top }}, \\
\tilde{\psi}_{\text {right-top }}=\chi^{(\tilde{\eta})} \otimes \chi^{(\tau)},
\end{gathered}
$$

where the tildes on the state vectors indicate that they are expressed in the basis of $\left(v_{1}, v_{2}\right)$ used in Eq. B8. A robust solution must be part of $\operatorname{ker}\left(\tilde{\sigma}_{1}+\mathrm{i} \gamma_{\text {right-top }} v_{y} \tilde{\sigma}_{2}\right)\left(\tau_{3}+\mathrm{i} \tau_{2}\right)$, and only exists if $\gamma_{\text {right-top }}=1 / v_{y}$. The positive-energy solution, at $E=J_{\mathrm{D}}$, is

$$
\tilde{\psi}_{\text {right-top }}=\left[\begin{array}{l}
1 \\
0
\end{array}\right]^{(\tilde{\sigma})} \otimes\left[\begin{array}{l}
1 \\
0
\end{array}\right]^{(\tau)},
$$

or in the original representation,

$$
\psi_{\text {right-top }}=\left[\begin{array}{l}
1 \\
0
\end{array}\right]^{(\sigma)} \otimes\left[\begin{array}{l}
1 \\
0
\end{array}\right]^{(\eta)} \otimes\left[\begin{array}{l}
1 \\
0
\end{array}\right]^{(\tau)} .
$$

Hence, this verifies the claim made in the main text: $\psi_{\text {top-right }}=\psi_{\text {right-top }}$, showing the top-edge and right-edge Hamiltonians share the same corner mode, as expected for a $2 \mathrm{D}$ HOT phase.

\section{Model II: tSOC model}

Owing to its similarity with the tBBH model, the tSOC model in the presence of a staggered Zeeman field $h_{\mathrm{s}}$ can be used to demonstrate the existence of corner states shared between the edge theories. We now elaborate on the discussion from the main text.

\section{a. Effective edge theories}

For concreteness, we assume $J_{2}>0$ and $D>0$ and study the gap closing that occurs when $J_{1}=J_{2}$ and $h_{\mathrm{s}}=h_{\mathrm{s}}^{(\mathrm{c})}$, at momentum $\mathbf{k}_{0}=(\pi, \pi / 2)$. The linearized Hamiltonian in the neighborhood of this point is

$$
\begin{gathered}
\tau_{3} H_{\mathrm{cont}}^{\mathrm{tSOC}}=J_{\mathrm{D}} \sigma_{0} \eta_{0} \tau_{3}-J\left(-\mathrm{i} \partial_{y}\right) \sigma_{0} \eta_{0}\left(\tau_{3}-\mathrm{i} \tau_{2}\right) \\
+\left(\frac{J_{1}-J_{2}}{2} \sigma_{1}-\frac{J_{2}}{2}\left(-\mathrm{i} \partial_{x}\right) \sigma_{2}\right) \eta_{0}\left(\tau_{3}-\mathrm{i} \tau_{2}\right) \\
-\sigma_{3} \eta_{2}\left(h_{\mathrm{s}} \tau_{0}-D\left(\tau_{3}-\mathrm{i} \tau_{2}\right)\right) \\
-\Gamma\left(-\mathrm{i} \partial_{y}\right) \sigma_{3} \eta_{1}\left(\tau_{3}-\mathrm{i} \tau_{2}\right) .
\end{gathered}
$$

This equation, with $J=0$, was reported in the main text in Eq. 34. As explained in the main text, we can analyze the model in the large- $J_{\mathrm{D}}$ limit; this way, mass terms analogous to those of Eq. 30 are easily found. In this limit, we can obtain an effective (Hermitian) Hamiltonian for the positive-energy states using a first-order SchriefferWolff transformation: the unperturbed Hamiltonian is $J_{\mathrm{D}} \sigma_{0} \eta_{0} \tau_{3}$, and the subspace of interest is made up of the four particle-like states of the unperturbed Hamiltonian, with the associated projector $\mathcal{P}=\sigma_{0} \eta_{0}\left(\tau_{0}+\tau_{3}\right) / 2$. The first-order effective Hamiltonian in $\mathcal{O}\left(1 / J_{\mathrm{D}}\right)$ becomes asymptotically exact in the large- $J_{\mathrm{D}}$ limit, and is simply obtained by the projection the full Hamiltonian $\tau_{3} H_{\text {cont }}^{\mathrm{tSOC}}$ onto the subspace of interest:

$$
\mathcal{P}\left(\tau_{3} H_{\mathrm{cont}}^{\mathrm{tSOC}}\right) \mathcal{P}=\tilde{H}_{\mathrm{cont}}^{\mathrm{tSOC}} \mathcal{P},
$$


where

$$
\begin{aligned}
\tilde{H}_{\mathrm{cont}}^{\mathrm{tSOC}}= & \left(J_{\mathrm{D}}-J\left(-\mathrm{i} \partial_{y}\right)\right) \sigma_{0} \eta_{0} \\
+ & \left(\frac{J_{1}-J_{2}}{2} \sigma_{1}-\frac{J_{2}}{2}\left(-\mathrm{i} \partial_{x}\right) \sigma_{2}\right) \eta_{0} \\
& -\sigma_{3}\left(\left(h_{\mathrm{s}}-D\right) \eta_{2}+\Gamma\left(-\mathrm{i} \partial_{y}\right) \eta_{1}\right) .
\end{aligned}
$$

In this limit, $h_{\mathrm{c}} \rightarrow D$, so the mass term is manifest: $m_{y}=h_{\mathrm{s}}-D$. We also let $m_{x}=\left(J_{1}-J_{2}\right) / 2, v_{x}=J_{2} / 2$, and $v_{y}=\Gamma$, so

$$
\begin{aligned}
\tilde{H}_{\mathrm{cont}}^{\mathrm{tSOC}}=\left(J_{\mathrm{D}}-J\left(-\mathrm{i} \partial_{y}\right)\right) \sigma_{0} \eta_{0} & \\
& +\left(m_{x} \sigma_{1}-v_{x}\left(-\mathrm{i} \partial_{x}\right) \sigma_{2}\right) \eta_{0} \\
& \quad-\sigma_{3}\left(m_{y} \eta_{2}+v_{y}\left(-\mathrm{i} \partial_{y}\right) \eta_{1}\right) .
\end{aligned}
$$

The effect of a nonzero $J$ is to tilt the dispersion along the $k_{y}$ direction. The outcome of the present calculation is qualitatively the same as long as the Dirac crossing is not inverted (i.e., as long as $|J|<\left|v_{y}\right|=|\Gamma|$ ), so we let $J=0$ for simplicity.

We create a domain wall along the top edge ( $y=0$ line) using a Jackiw-Rebbi ansatz:

$$
\psi_{q_{x}}(x, y)=\mathrm{e}^{-\gamma_{\text {top }} \int_{0}^{y} \mathrm{~d} \tilde{y} m_{y}(\tilde{y})} \mathrm{e}^{\mathrm{i} x q_{x}} \psi_{\text {top }}\left(q_{x}\right) .
$$

Independently of the details of $m_{y}(y)$, the state $\psi_{q_{x}}(x, y)$ must be an eigenstate of $\tilde{H}_{\mathrm{cont}}^{\mathrm{tSOC}}$, meaning

$$
\tilde{H}_{\text {cont }}^{\mathrm{tSOC}} \psi_{q_{x}}(x, y)=E_{q_{x}} \psi_{q_{x}}(x, y) .
$$

Hence, $\psi_{\text {top }}\left(q_{x}\right)$ must be part of $\operatorname{ker}\left(\sigma_{3}\left(\eta_{2}+\mathrm{i} \gamma_{\text {top }} v_{y} \eta_{1}\right)\right)$. Solutions only arise if $\gamma_{\text {top }}=1 / v_{y}$, and are of the form

$$
\psi_{\text {top }}\left(q_{x}\right)=\chi_{q_{x}}^{(\sigma)} \otimes\left[\begin{array}{l}
0 \\
1
\end{array}\right]^{(\eta)},
$$

where $\chi_{q_{x}}^{(\sigma)}$ is an arbitrary two-component vector. The effective top-edge Hamiltonian is then

$$
\tilde{H}_{\text {top }}^{\mathrm{tSOC}}=J_{\mathrm{D}} \sigma_{0}+m_{x} \sigma_{1}-v_{x}\left(-\mathrm{i} \partial_{x}\right) \sigma_{2} .
$$

We next create a domain wall along the right edge ( $x=0$ line) using the following Jackiw-Rebbi ansatz:

$$
\begin{gathered}
\psi_{q_{y}}(x, y)=\mathrm{e}^{-\gamma_{\mathrm{right}} \int_{0}^{x} \mathrm{~d} \tilde{x} m_{x}(\tilde{x})} \mathrm{e}^{\mathrm{i} y q_{y}} \psi_{\text {right }}\left(q_{y}\right), \\
\tilde{H}_{\text {cont }}^{\mathrm{tSOC}} \psi_{q_{y}}(x, y)=E_{q_{y}} \psi_{q_{y}}(x, y) .
\end{gathered}
$$

Nontrivial solutions arise only if $\gamma_{\text {right }}=1 / v_{x}$, and are such that $\psi_{\text {right }}\left(q_{y}\right) \in \operatorname{ker}\left(\left(\sigma_{1}-\mathrm{i} \sigma_{2}\right) \eta_{0}\right)$, meaning

$$
\psi_{\text {right }}\left(q_{y}\right)=\left[\begin{array}{l}
0 \\
1
\end{array}\right]^{(\sigma)} \otimes \chi_{q_{y}}^{(\eta)} .
$$

This implies the effective Hamiltonian for the right edge is

$$
\tilde{H}_{\text {right }}^{\mathrm{tSOC}}=J_{\mathrm{D}} \eta_{0}+m_{y} \eta_{2}+v_{y}\left(-\mathrm{i} \partial_{y}\right) \eta_{1} .
$$

\section{b. $\quad$ Shared corner modes}

We now verify the existence of shared corner modes by introducing domain walls in the edge Hamiltonians $\tilde{H}_{\text {top }}^{\text {tSOC }}$ and $\tilde{H}_{\text {right }}^{\mathrm{tSOC}}$.

To find the corner mode originating from the top-edge Hamiltonian, we use the ansatz

$$
\begin{gathered}
\psi_{\text {top }}(x)=\mathrm{e}^{-\gamma_{\text {top-right }} \int_{0}^{x} \mathrm{~d} \tilde{x} m_{x}(\tilde{x})} \psi_{\text {top-right }}, \\
\psi_{\text {top-right }}=\chi^{(\sigma)} \otimes\left[\begin{array}{l}
0 \\
1
\end{array}\right]^{(\eta)} .
\end{gathered}
$$

Such a solution exists if $\gamma_{\text {top-right }}=1 / v_{x}$ and is part of $\operatorname{ker}\left(\sigma_{1}-\mathrm{i} \sigma_{2}\right)$, leading to the following solution at energy $E=J_{\mathrm{D}}$ :

$$
\psi_{\text {top-right }}=\left[\begin{array}{l}
0 \\
1
\end{array}\right]^{(\sigma)} \otimes\left[\begin{array}{l}
0 \\
1
\end{array}\right]^{(\eta)}
$$

The corner mode originating from the right-edge Hamiltonian is found using the ansatz

$$
\begin{gathered}
\psi_{\text {right }}(y)=\mathrm{e}^{-\gamma_{\text {right-top }} \int_{0}^{y} \mathrm{~d} \tilde{y} m_{y}(\tilde{y})} \psi_{\text {right-top }}, \\
\psi_{\text {right-top }}=\left[\begin{array}{l}
0 \\
1
\end{array}\right]^{(\sigma)} \otimes \chi^{(\eta)}
\end{gathered}
$$

A solution arises if $\gamma_{\text {right-top }}=1 / v_{y}$ and is part of $\operatorname{ker}\left(\eta_{2}+\right.$ $\left.\mathrm{i} \eta_{1}\right)$. Specifically,

$$
\psi_{\text {right-top }}=\left[\begin{array}{l}
0 \\
1
\end{array}\right]^{(\sigma)} \otimes\left[\begin{array}{l}
0 \\
1
\end{array}\right]^{(\eta)},
$$

at energy $E=J_{\mathrm{D}}$.

Comparing $\psi_{\text {top-right }}$ with $\psi_{\text {right-top }}$ we find that they are identical, thus confirming that the corner mode is shared between the top and right edges.

\section{Appendix C: Invariance under mirror-like symmetries}

In this section, we obtain the symmetry-allowed spin interactions for the two models above. We approach this by considering the symmetry-allowed forms of the tensors $\mathbb{J}^{\mathbf{r}, \mathbf{r}^{\prime}}$ for all possible bilinear spin interactions $\mathbf{S}_{\mathbf{r}}^{\top} \mathbb{J}^{\mathbf{r}, \mathbf{r}^{\prime}} \mathbf{S}_{\mathbf{r}^{\prime}}$ between sites $\mathbf{r}$ and $\mathbf{r}^{\prime}$. In both cases, we assume the only interaction between bottom and top layers is the strong antiferromagnetic exchange $J_{\mathrm{D}}$ within each dimer. Hence, we may drop the layer index and treat each layer individually for the symmetry analysis.

\section{Model I: tBBH model}

A priori, there are eight bilinear spin interactions to consider, four of which are intra-unit cell and four, interunit cell. We write the transformations in terms of the 
matrices $R_{x}=\operatorname{diag}(1,-1,-1)$ and $R_{y}=\operatorname{diag}(-1,1,-1)$. For notational brevity, we define $\tilde{M}_{x}:=\tilde{C}_{2}^{z} \circ M_{x}$. We discuss the symmetry transformations of the interaction tensors on the horizontal and vertical bonds below.

\section{a. Intracell bonds}

Horizontal bonds The intracell interaction tensors $\mathbb{J}_{\text {intra }}^{3,1}$ (connecting dimers 3 and 1 in Fig. 1(a) and (b)) and $\mathbb{J}_{\text {intra }}^{2,4}$ (connecting dimers 2 and 4 ) transform as follows under the symmetries:

$$
\begin{gathered}
\mathbf{S}_{\mathbf{R}, 3}^{\top} \mathbb{J}_{\text {intra }}^{3,1} \mathbf{S}_{\mathbf{R}, 1} \stackrel{\tilde{M}_{x}}{\rightarrow} \mathbf{S}_{\mathbf{R}^{\prime}, 1}^{\top} R_{y}^{\top} \mathbb{J}_{\text {intra }}^{3,1} R_{y} \mathbf{S}_{\mathbf{R}^{\prime}, 3} \\
=\mathbf{S}_{\mathbf{R}^{\prime}, 3}^{\top} R_{y}^{\top} \mathbb{J}_{\text {intra }}^{3,1} R_{y} \mathbf{S}_{\mathbf{R}^{\prime}, 1} ; \quad(\mathrm{C} 1 \mathrm{a}) \\
\mathbf{S}_{\mathbf{R}, 2}^{\top} \mathbb{J}_{\text {intra }}^{2,4} \mathbf{S}_{\mathbf{R}, 4} \stackrel{\tilde{M}_{x}}{\rightarrow} \mathbf{S}_{\mathbf{R}^{\prime}, 4}^{\top} R_{x}^{\top} \mathbb{J}_{\text {intra }}^{2,4} R_{x} \mathbf{S}_{\mathbf{R}^{\prime}, 2} \\
=\mathbf{S}_{\mathbf{R}^{\prime}, 2}^{\top} R_{x}^{\top} \mathbb{J}_{\text {intra }}^{2,4} R_{x} \mathbf{S}_{\mathbf{R}^{\prime}, 4} ; \quad(\mathrm{C} 1 \mathrm{~b}) \\
\mathbf{S}_{\mathbf{R}, 3}^{\top} \mathbb{J}_{\text {intra }}^{3,1} \mathbf{S}_{\mathbf{R}, 1}+\mathbf{S}_{\mathbf{R}, 2}^{\top} \mathbb{J}_{\text {intra }}^{2,4} \mathbf{S}_{\mathbf{R}, 4} \\
\stackrel{M_{y}}{\rightarrow} \mathbf{S}_{\mathbf{R}^{\prime \prime}, 2}^{\top} R_{y}^{\top} \mathbb{J}_{\text {intra }}^{3,1} R_{y} \mathbf{S}_{\mathbf{R}^{\prime \prime}, 4}+\mathbf{S}_{\mathbf{R}^{\prime \prime}, 3}^{\top} R_{y}^{\top} \mathbb{J}_{\text {intra }}^{2,4} R_{y} \mathbf{S}_{\mathbf{R}^{\prime \prime}, 1} .
\end{gathered}
$$

Hence, the symmetries constrain these exchange tensors as follows:

$$
\begin{gathered}
\mathbb{J}_{\text {intra }}^{3,1} \stackrel{\tilde{M}_{x}}{=} R_{y}^{\top} \mathbb{J}_{\text {intra }}^{3,1}{ }^{\top} R_{y} ; \\
\mathbb{J}_{\text {intra }}^{2,4} \stackrel{\tilde{M}_{x}}{=} R_{x}^{\top} \mathbb{J}_{\text {intra }}^{2,4} R_{x} ; \\
\mathbb{J}_{\text {intra }}^{3,1} \stackrel{M_{y}}{=} R_{y}^{\top} \mathbb{J}_{\text {intra }}^{2,4} R_{y} ; \quad \mathbb{J}_{\text {intra }}^{2,4} \stackrel{M_{y}}{=} R_{y}^{\top} \mathbb{J}_{\text {intra }}^{3,1} R_{y} .
\end{gathered}
$$

This leads to the following explicit structure for the tensors:

$$
\mathbb{J}_{\text {intra }}^{3,1}=\left[\begin{array}{rrr}
a_{11} & a_{12} & \\
-a_{12} & a_{22} & \\
& & a_{33}
\end{array}\right], \mathbb{J}_{\text {intra }}^{2,4}=\left[\begin{array}{rrr}
a_{11} & -a_{12} & \\
a_{12} & a_{22} & \\
& & a_{33}
\end{array}\right] .
$$

In our model, we have $a_{12}=0$ and $a_{11}=a_{22}=a_{33}=J_{1}^{x}$, which is compatible with the symmetry constraints.

Vertical bonds The intracell interaction tensors $\mathbb{J}_{\text {intra }}^{2,3}$ (connecting dimers 2 and 3 in Fig. $1(b)$ ) and $J_{\text {intra }}^{4,1}$ (connecting dimers 4 and 1 ) transform as follows:

$$
\begin{gathered}
\mathbf{S}_{\mathbf{R}, 2}^{\top} \mathbb{J}_{\text {intra }}^{2,3} \mathbf{S}_{\mathbf{R}, 3} \stackrel{M_{y}}{\rightarrow} \mathbf{S}_{\mathbf{R}^{\prime}, 3}^{\top} R_{y}^{\top} \mathbb{J}_{\text {intra }}^{2,3} R_{y} \mathbf{S}_{\mathbf{R}^{\prime}, 2} \\
=\mathbf{S}_{\mathbf{R}^{\prime}, 2}^{\top} R_{y}^{\top} \mathbb{J}_{\text {intra }}^{2,3} R_{y} \mathbf{S}_{\mathbf{R}^{\prime}, 3} ; \quad(\mathrm{C} 4 \mathrm{a}) \\
\mathbf{S}_{\mathbf{R}, 4}^{\top} \mathbb{J}_{\text {intra }}^{4,1} \mathbf{S}_{\mathbf{R}, 1} \stackrel{M_{y}}{\rightarrow} \mathbf{S}_{\mathbf{R}^{\prime}, 1}^{\top} R_{y}^{\top} \mathbb{J}_{\text {intra }}^{4,1} R_{y} \mathbf{S}_{\mathbf{R}^{\prime}, 4} \\
=\mathbf{S}_{\mathbf{R}^{\prime}, 4}^{\top} R_{y}^{\top} \mathbb{J}_{\text {intra }}^{4,1}{ }^{\top} R_{y} \mathbf{S}_{\mathbf{R}^{\prime}, 1} ; \quad(\mathrm{C} 4 \mathrm{~b}) \\
\mathbf{S}_{\mathbf{R}, 2}^{\top} \mathbb{J}_{\text {intra }}^{2,3} \mathbf{S}_{\mathbf{R}, 3}+\mathbf{S}_{\mathbf{R}, 4}^{\top} \mathbb{J}_{\text {intra }}^{4,1} \mathbf{S}_{\mathbf{R}, 1} \\
\stackrel{\tilde{M}_{x}}{\rightarrow} \mathbf{S}_{\mathbf{R}^{\prime \prime}, 4}^{\top} R_{x}^{\top} \mathbb{J}_{\text {intra }}^{2,3} R_{y} \mathbf{S}_{\mathbf{R}^{\prime \prime}, 1}+\mathbf{S}_{\mathbf{R}^{\prime \prime}, 2}^{\top} R_{x}^{\top} \mathbb{J}_{\text {intra }}^{4,1} R_{y} \mathbf{S}_{\mathbf{R}^{\prime \prime}, 3} .
\end{gathered}
$$

Hence, the symmetry constraints on these tensors are

$$
\begin{gathered}
\mathbb{J}_{\text {intra }}^{2,3} \stackrel{\stackrel{M_{y}}{=}}{=} R_{y}^{\top} \mathbb{J}_{\text {intra }}^{2,3} R_{y} ; \\
\mathbb{J}_{\text {intra }}^{4,1} \stackrel{M_{y}}{=} R_{y}^{\top} \mathbb{J}_{\text {intra }}^{4,1} R_{y} ; \\
\mathbb{J}_{\text {intra }}^{2,3} \stackrel{\tilde{M}_{x}}{=} R_{x}^{\top} \mathbb{J}_{\text {intra }}^{4,1} R_{y} ; \quad \mathbb{J}_{\text {intra }}^{4,1} \stackrel{\tilde{M}_{x}}{=} R_{x}^{\top} \mathbb{J}_{\text {intra }}^{2,3} R_{y},
\end{gathered}
$$

and their explicit structure is

$$
\mathbb{J}_{\text {intra }}^{2,3}=\left[\begin{array}{rrr}
-b_{11} & b_{12} & \\
-b_{12} & -b_{22} & \\
& & b_{33}
\end{array}\right], \mathbb{J}_{\text {intra }}^{4,1}=\left[\begin{array}{rrr}
b_{11} & b_{12} & \\
-b_{12} & b_{22} & \\
& & b_{33}
\end{array}\right] \text {. }
$$

In our model, we have $b_{12}=0$ and $b_{11}=b_{22}=b_{33}=J_{1}^{y}$, making it compatible with the symmetry constraints.

\section{b. Intercell bonds}

The symmetry constraints for the tensors on the intercell bonds can also be derived using the previous analysis. At the end of the analysis, we find that the horizontal intercell tensors $\mathbb{J}_{\text {inter }}^{1,3}$ and $\mathbb{J}_{\text {inter }}^{4,2}$ have the same form (Eq. C3) as the horizontal intracell tensors $\mathbb{J}_{\text {intra }}^{3,1}$ and $\mathbb{J}_{\text {intra }}^{2,4}$, respectively. Likewise, the vertical intercell tensors $\mathbb{J}_{\text {inter }}^{1,4}$ and $\mathbb{J}_{\text {inter }}^{3,2}$ have the same structure (Eq. C6) as the tensors $\mathbb{J}_{\text {intra }}^{4,1}$ and $\mathbb{J}_{\text {intra }}^{2,3}$, respectively. Specifically,

$$
\mathbb{J}_{\text {inter }}^{1,3}=\left[\begin{array}{rrr}
c_{11} & c_{12} & \\
-c_{12} & c_{22} & \\
& & c_{33}
\end{array}\right], \quad \mathbb{J}_{\text {inter }}^{4,2}=\left[\begin{array}{rrr}
c_{11} & -c_{12} & \\
c_{12} & c_{22} & \\
& & c_{33}
\end{array}\right],
$$

$\mathbb{J}_{\text {inter }}^{3,2}=\left[\begin{array}{rrr}-d_{11} & d_{12} & \\ -d_{12} & -d_{22} & \\ & & d_{33}\end{array}\right], \quad \mathbb{J}_{\text {inter }}^{1,4}=\left[\begin{array}{rrr}d_{11} & d_{12} & \\ -d_{12} & d_{22} & \\ & & d_{33}\end{array}\right]$.

For the present model, we have $c_{12}=0, c_{11}=c_{22}=$ $c_{33}=J_{2}^{x}, d_{12}=0$, and $d_{11}=d_{22}=d_{33}=J_{2}^{y}$, making the model compatible with the symmetry constraints for the intercell bonds as well.

\section{Model II: tSOC model}

In the tSOC model, we consider four bilinear spin interactions, two of which are horizontal and two, vertical. To describe the transformations, we define the matrices

$$
R_{x}=\left[\begin{array}{lll}
1 & & \\
& -1 & \\
& & -1
\end{array}\right], \quad \tilde{R}_{y}=\left[\begin{array}{lll}
0 & 1 & \\
1 & 0 & \\
& & -1
\end{array}\right] .
$$

\section{a. Horizontal bonds}

The interaction tensors $\mathbb{J}_{\text {intra }}^{1,2}$ (between $\mathbf{S}_{\mathbf{R}, 1}$ and $\mathbf{S}_{\mathbf{R}, 2}$; see Fig. 1(c) and (d)) and $\mathbb{J}_{\text {inter }}^{2,1}$ (between $\mathbf{S}_{\mathbf{R}, 2}$ and 
$\left.\mathbf{S}_{\mathbf{R}+\hat{x}, 1}\right)$ transform as follows under the symmetries:

$$
\begin{array}{r}
\mathbf{S}_{\mathbf{R}, 1}^{\top} \mathbb{J}_{\text {intra }}^{1,2} \mathbf{S}_{\mathbf{R}, 2} \stackrel{M_{x}}{\rightarrow} \mathbf{S}_{\mathbf{R}^{\prime}, 2}^{\top} R_{x}^{\top} \mathbb{J}_{\text {intra }}^{1,2} R_{x} \mathbf{S}_{\mathbf{R}^{\prime}, 1} \\
=\mathbf{S}_{\mathbf{R}^{\prime}, 1}^{\top} R_{x}^{\top} \mathbb{J}_{\text {intra }}^{1,2}{ }^{\top} R_{x} \mathbf{S}_{\mathbf{R}^{\prime}, 2}, \\
\mathbf{S}_{\mathbf{R}, 1}^{\top} \mathbb{J}_{\text {intra }}^{1,2} \mathbf{S}_{\mathbf{R}, 2} \stackrel{\tilde{M}_{y}}{\rightarrow} \mathbf{S}_{\mathbf{R}^{\prime \prime}, 1}^{\top} \tilde{R}_{y}^{\top} \mathbb{J}_{\text {intra }}^{1,2} \tilde{R}_{y} \mathbf{S}_{\mathbf{R}^{\prime \prime}, 2} ; \\
\mathbf{S}_{\mathbf{R}, 2}^{\top} \mathbb{J}_{\text {inter }}^{2,1} \mathbf{S}_{\mathbf{R}+\hat{x}, 1} \stackrel{M_{x}}{\rightarrow} \mathbf{S}_{\mathbf{R}^{\prime}, 1}^{\top} R_{x}^{\top} \mathbb{J}_{\text {inter }}^{2,1} \mathbf{S}_{\mathbf{R}^{\prime}-\hat{x}, 2} \\
=\mathbf{S}_{\mathbf{R}^{\prime}, 2}^{\top} R_{x}^{\top} \mathbb{J}_{\text {inter }}^{2,1} \mathbf{S}_{\mathbf{R}^{\prime}+\hat{x}, 1}, \\
\mathbf{S}_{\mathbf{R}, 2}^{\top} \mathbb{J}_{\text {inter }}^{2,1} \mathbf{S}_{\mathbf{R}+\hat{x}, 1} \stackrel{\tilde{M}_{y}}{\rightarrow} \mathbf{S}_{\mathbf{R}^{\prime \prime}, 2}^{\top} \tilde{R}_{y}^{\top} \mathbb{J}_{\text {inter }}^{2,1} \tilde{R}_{y} \mathbf{S}_{\mathbf{R}^{\prime \prime}+\hat{x}, 1},
\end{array}
$$

constraining these exchange tensors as follows:

$$
\begin{aligned}
& \mathbb{J}_{\text {intra }}^{1,2} \stackrel{M_{x}}{=} R_{x}^{\top} \mathbb{J}_{\text {intra }}^{1,2} R_{x}, \quad \mathbb{J}_{\text {intra }}^{1,2} \stackrel{\tilde{M}_{y}}{=} \tilde{R}_{y}^{\top} \mathbb{J}_{\text {intra }}^{1,2} \tilde{R}_{y} ; \\
& \mathbb{J}_{\text {inter }}^{2,1} \stackrel{M_{x}}{=} R_{x}^{\top} \mathbb{J}_{\text {inter }}^{2,1} R_{x}, \quad \mathbb{J}_{\text {inter }}^{2,1} \stackrel{\tilde{M}_{y}}{=} \tilde{R}_{y}^{\top} \mathbb{J}_{\text {inter }}^{2,1} \tilde{R}_{y} \text {. }
\end{aligned}
$$

The explicit form of the interaction tensors is

$$
\mathbb{J}_{\text {intra }}^{1,2}=\operatorname{diag}\left(a_{11}, a_{11}, a_{33}\right), \quad \mathbb{J}_{\text {inter }}^{2,1}=\operatorname{diag}\left(b_{11}, b_{11}, b_{33}\right) .
$$

In our model, $a_{11}=a_{33}=J_{1}$ and $b_{11}=b_{33}=J_{2}$, making it exactly compatible with the symmetry conditions.

\section{b. $\quad$ Vertical bonds}

The interaction terms $\mathbb{J}_{\text {inter }}^{1,1}$ (between $\mathbf{S}_{\mathbf{R}, 1}$ and $\mathbf{S}_{\mathbf{R}+\hat{y}, 1}$; see Fig. 1(c) and (d)) and $\mathbb{J}_{\text {inter }}^{2,2}$ (between $\mathbf{S}_{\mathbf{R}, 2}$ and $\mathbf{S}_{\mathbf{R}+\hat{y}, 2}$ ) transform as follows under the symmetries.

$$
\begin{aligned}
& \mathbf{S}_{\mathbf{R}, 1}^{\top} \mathbb{J}_{\text {inter }}^{1,1} \mathbf{S}_{\mathbf{R}+\hat{y}, 1} \stackrel{\tilde{M}_{y}}{\rightarrow} \mathbf{S}_{\mathbf{R}^{\prime \prime}, 1}^{\top} \tilde{R}_{y}^{\top} \mathbb{J}_{\text {inter }}^{1,1} \tilde{R}_{y} \mathbf{S}_{\mathbf{R}^{\prime \prime}-\hat{y}, 1} \\
& =\mathbf{S}_{\mathbf{R}^{\prime \prime}-\hat{y}, 1}^{\top} \tilde{R}_{y}^{\top} \mathbb{J}_{\text {inter }}^{1,1}{ }^{\top} \tilde{R}_{y} \mathbf{S}_{\mathbf{R}^{\prime \prime}, 1} \\
& =\mathbf{S}_{\mathbf{R}^{\prime \prime}, 1}^{\top} \tilde{R}_{y}^{\top} \mathbb{J}_{\text {inter }}^{1,1}{ }^{\top} \tilde{R}_{y} \mathbf{S}_{\mathbf{R}^{\prime \prime}+\hat{y}, 1} ; \\
& \mathbf{S}_{\mathbf{R}, 2}^{\top} \mathbb{J}_{\text {inter }}^{2,2} \mathbf{S}_{\mathbf{R}+\hat{y}, 2} \stackrel{\tilde{M}_{y}}{\rightarrow} \mathbf{S}_{\mathbf{R}^{\prime \prime}, 2}^{\top} \tilde{R}_{y}^{\top} \mathbb{J}_{\text {inter }}^{2,2} \tilde{R}_{y} \mathbf{S}_{\mathbf{R}^{\prime \prime}-\hat{y}, 2} \\
& =\mathbf{S}_{\mathbf{R}^{\prime \prime}-\hat{y}, 2}^{\top} \tilde{R}_{y}^{\top} \mathbb{J}_{\text {inter }}^{2,2}{ }^{\top} \tilde{R}_{y} \mathbf{S}_{\mathbf{R}^{\prime \prime}, 2} \\
& =\mathbf{S}_{\mathbf{R}^{\prime \prime}, 2}^{\top} \tilde{R}_{y}^{\top} \mathbb{J}_{\text {inter }}^{2,2}{ }^{\top} \tilde{R}_{y} \mathbf{S}_{\mathbf{R}^{\prime \prime}+\hat{y}, 2} ; \\
& \mathbf{S}_{\mathbf{R}, 1}^{\top} \mathbb{J}_{\text {inter }}^{1,1} \mathbf{S}_{\mathbf{R}+\hat{y}, 1}+\mathbf{S}_{\mathbf{R}, 2}^{\top} \mathbb{J}_{\text {inter }}^{2,2} \mathbf{S}_{\mathbf{R}+\hat{y}, 2} \\
& \stackrel{M_{x}}{\rightarrow} \mathbf{S}_{\mathbf{R}^{\prime}, 2}^{\top} R_{x}^{\top} \mathbb{J}_{\text {inter }}^{1,1} R_{x} \mathbf{S}_{\mathbf{R}^{\prime}+\hat{y}, 2}+\mathbf{S}_{\mathbf{R}^{\prime}, 1}^{\top} R_{x}^{\top} \mathbb{J}_{\text {inter }}^{2,2} R_{x} \mathbf{S}_{\mathbf{R}^{\prime}+\hat{y}, 1} \text {. }
\end{aligned}
$$

(C13c)

The exchange tensors must obey the following symmetry constraints.

$$
\begin{gathered}
\mathbb{J}_{\text {inter }}^{1,1} \stackrel{M_{x}}{=} R_{x}^{\top} \mathbb{J}_{\text {inter }}^{2,2} R_{x}, ; \quad \mathbb{J}_{\text {inter }}^{2,2} \stackrel{M_{x}}{=} R_{x}^{\top} \mathbb{J}_{\text {inter }}^{1,1} R_{x} ; \\
\mathbb{J}_{\text {inter }}^{1,1} \stackrel{\stackrel{\tilde{M}_{y}}{=}}{=} \tilde{R}_{y}^{\top} \mathbb{J}_{\text {inter }}^{1,1}{ }^{\top} \tilde{R}_{y} ; \\
\mathbb{J}_{\text {inter }}^{2,2} \stackrel{\stackrel{\tilde{M}_{y}}{=}}{=} \tilde{R}_{y}^{\top} \mathbb{J}_{\text {inter }}^{2,2}{ }^{\top} \tilde{R}_{y}
\end{gathered}
$$

These constraints yield the following explicit form for the interaction tensors.

$$
\mathbb{J}_{\text {inter }}^{1,1}=\left[\begin{array}{rrr}
c_{11} & -c_{12} & \\
-c_{21} & c_{11} & \\
& & c_{33}
\end{array}\right], \quad \mathbb{J}_{\text {inter }}^{2,2}=\left[\begin{array}{lll}
c_{11} & c_{12} & \\
c_{21} & c_{11} & \\
& & c_{33}
\end{array}\right]
$$

In our model, $c_{11}=c_{33}=J, c_{12}=\Gamma+D$ and $c_{21}=\Gamma-D$, making it exactly compatible with the symmetries.

\section{c. Zeeman field}

As stated in the main text, the symmetries $M_{x}$ and $\tilde{M}_{y}$ do not allow a Zeeman field. However, as we shall see, certain Zeeman fields are allowed if one or both symmetry operations are modified to include time reversal. Note that the other interactions in the spin Hamiltonian are invariant under time reversal because they are all bilinear in spins with real coefficients.

We consider a general (translation-invariant) Zeeman coupling term, $\sum_{a \in\{1,2\}} \mathbf{B}_{a}^{\top} \mathbf{S}_{\mathbf{R}, a}$, where $\mathbf{B}_{a}=$ $\left[B_{a}^{x}, B_{a}^{y}, B_{a}^{z}\right]^{\top}$. Under the operation $\tilde{M}_{y}$, the Zeeman term transforms as

$$
\mathbf{B}_{a}^{\top} \mathbf{S}_{\mathbf{R}, a} \stackrel{\tilde{M}_{y}}{\rightarrow} \mathbf{B}_{a}^{\top} \tilde{R}_{y} \mathbf{S}_{\mathbf{R}^{\prime \prime}, a},
$$

leading to the constraint $\mathbf{B}_{a} \stackrel{\tilde{M}_{y}}{=} \tilde{R}_{y}^{\top} \mathbf{B}_{a}$. This constraint implies that $\mathbf{B}_{a}$ must be an eigenvector of $\tilde{R}_{y}^{\top}$ with eigenvalue 1 . The only such eigenvector is $[1,1,0]^{\top}$, meaning $B_{a}^{x}=B_{a}^{y}$ and $B_{a}^{z}=0$ for $a \in\{1,2\}$.

On the other hand, under $M_{x}$, the transformation proceeds as

$$
\mathbf{B}_{1}^{\top} \mathbf{S}_{\mathbf{R}, 1}+\mathbf{B}_{2}^{\top} \mathbf{S}_{\mathbf{R}, 2} \stackrel{M_{x}}{\rightarrow} \mathbf{B}_{1}^{\top} R_{x} \mathbf{S}_{\mathbf{R}^{\prime}, 2}+\mathbf{B}_{2}^{\top} R_{x} \mathbf{S}_{\mathbf{R}^{\prime}, 1},
$$

giving rise to the constraint $\mathbf{B}_{1} \stackrel{M_{x}}{=} R_{x}^{\top} \mathbf{B}_{2}, \mathbf{B}_{2} \stackrel{M_{x}}{=} R_{x}^{\top} \mathbf{B}_{1}$. Explicitly, this implies $\left(B_{1}^{x}, B_{1}^{y}, B_{1}^{z}\right)=\left(B_{2}^{x},-B_{2}^{y},-B_{2}^{z}\right)$.

Under the modified operation $\mathcal{T} \circ \tilde{M}_{y}$, the Zeeman terms transform as

$$
\mathbf{B}_{a}^{\top} \mathbf{S}_{\mathbf{R}, a} \stackrel{\mathcal{T} \circ \tilde{M}_{y}}{\rightarrow}-\mathbf{B}_{a}^{\top} \tilde{R}_{y} \mathbf{S}_{\mathbf{R}^{\prime \prime}, a}
$$

leading to the constraint $\mathbf{B}_{a} \stackrel{\mathcal{T} \circ \tilde{M}_{y}}{=}-\tilde{R}_{y}^{\top} \mathbf{B}_{a}$. Since the eigenvectors of $\tilde{R}_{y}^{\top}$ with eigenvalue -1 are $[1,-1,0]^{\top}$ and $[0,0,1]^{\top}$, this constraint forces $B_{a}^{x}=-B_{a}^{y}$ and leaves $B_{a}^{z}$ unconstrained, where $a \in\{1,2\}$.

Finally, the spins transform as follows under $\mathcal{T} \circ M_{x}$ :

$$
\mathbf{B}_{1}^{\top} \mathbf{S}_{\mathbf{R}, 1}+\mathbf{B}_{2}^{\top} \mathbf{S}_{\mathbf{R}, 2} \stackrel{\mathcal{T} \circ M_{x}}{\rightarrow}-\mathbf{B}_{1}^{\top} R_{x} \mathbf{S}_{\mathbf{R}^{\prime}, 2}-\mathbf{B}_{2}^{\top} R_{x} \mathbf{S}_{\mathbf{R}^{\prime}, 1},
$$

This gives rise to the constraint $\mathbf{B}_{1} \stackrel{M_{x}}{=}-R_{x}^{\top} \mathbf{B}_{2}, \mathbf{B}_{2} \stackrel{M_{x}}{=}$ $-R_{x}^{\top} \mathbf{B}_{1}$, and implies $\left(B_{1}^{x}, B_{1}^{y}, B_{1}^{z}\right)=\left(-B_{2}^{x}, B_{2}^{y}, B_{2}^{z}\right)$

The symmetry-allowed Zeeman terms for the different combination of symmetries are summarized in Table I, and verify the assertions made in the main text. 


\begin{tabular}{c||c|c} 
& $\tilde{M}_{y}$ & $\mathcal{T} \circ \tilde{M}_{y}$ \\
\hline \hline$M_{x}$ & $\mathbf{B}_{1}=\mathbf{B}_{2}=0$ & $\mathbf{B}_{1}=-\mathbf{B}_{2}=h_{\mathrm{s}} \hat{z}$ \\
\hline $\mathcal{T} \circ M_{x}$ & $\mathbf{B}_{1}=\mathbf{B}_{2}=0$ & $\mathbf{B}_{1}=+\mathbf{B}_{2}=h \hat{z}$
\end{tabular}

TABLE I. Symmetry constraints on Zeeman field.

\section{Implementation on triplons}

Any symmetry of the spin Hamiltonian that maps one dimer to another has a representation in the triplon Hamiltonian.

a. Unitary transformations If a symmetry $g$ transforms spins such that

$$
S_{i, \ell}^{\alpha} \stackrel{g}{\rightarrow} \sum_{\beta} T_{\alpha \beta} S_{i^{\prime}, \ell^{\prime}}^{\beta}
$$

for some $\mathrm{SO}(3)$ matrix $T$, its transformation on the triplon operators is

$$
t_{i, \alpha} \rightarrow\left\{\begin{array}{rl}
\sum_{\beta} T_{\alpha \beta} t_{i^{\prime}, \beta} & \text { if } \ell=\ell^{\prime} \\
-\sum_{\beta} T_{\alpha \beta} t_{i^{\prime}, \beta} & \text { if } \ell \neq \ell^{\prime}
\end{array} .\right.
$$

For the symmetries discussed in Sec. C 1 and C 2, the $\ell=\ell^{\prime}$ case in the above equation applies. Using this prescription and the transformations for the spins, it is straightforward to find the representations given in Eqs. 20 and 24 .

b. Antiunitary transformations Given the definition of Eq. 5 , the real-space triplon operators $t_{\mathbf{r} \alpha}$ are invariant under time reversal $\mathcal{T}$; hence, the action of time reversal on a real-space triplon Hamiltonian (e.g. Eqs. 6 and 8) is simply to complex-conjugate the coefficients [46]. From this, we see that the implementation of an antiunitary symmetry transformation $\mathcal{T} \circ g$, where $g$ is a unitary transformation, is the same as that of $g$ but with an additional (left-multiplied) complex conjugation $K$. For example, the transformations $\mathcal{T} \circ M_{x}$ and $\mathcal{T} \circ \tilde{M}_{y}=\mathcal{T} \circ C_{4}^{z} \circ M_{y}$ of the tSOC model have the following implementations:

$$
\begin{aligned}
& \mathcal{T} \circ M_{x} \equiv K \sigma_{1} \eta_{3} \tau_{0}, \\
& \mathcal{T} \circ \tilde{M}_{y} \equiv K \sigma_{0} \eta_{1} \tau_{0},
\end{aligned}
$$

\section{c.f. Eq. 24.}

Apart from Zeeman terms, the coefficients in real-space triplon Hamiltonians (e.g. Eqs. 6 and 8) are purely real, so the representation of $\mathcal{T} \circ g$, with its additional complex conjugation $K$, has precisely the same effect as the representation of $g$. This is a manifestation of the fact that symmetries symmetries $g$ and $\mathcal{T} \circ g$ are equivalent for bilinear spin interactions.

In the analysis of the tSOC model above, we found that the staggered Zeeman field configuration is compatible with symmetries $M_{x}$ and $\mathcal{T} \circ \tilde{M}_{y}$, while the uniform field configuration is compatible with $\mathcal{T} \circ M_{x}$ and $\mathcal{T} \circ \tilde{M}_{y}$. In the associated real-space triplon Hamiltonian, the Zeeman coupling enters in the intra-unit cell coupling matrix $M_{0}$ of Eq. 8 in the form of an additional term $-h_{\mathrm{s}} \sigma_{3} \eta_{2} \tau_{3}$ (staggered field) or $-h \sigma_{0} \eta_{2} \tau_{3}$ (uniform field). Using to the representations of Eqs. 24 and C22, it is easy to see that the Zeeman terms remain invariant in the following way. For the staggered field and the symmetries $M_{x}$ and $\mathcal{T} \circ \tilde{M}_{y}$, we have

$$
\begin{aligned}
\left(\sigma_{1} \eta_{3} \tau_{0}\right)^{-1}\left(-h_{\mathrm{s}} \sigma_{3} \eta_{2} \tau_{3}\right)\left(\sigma_{1} \eta_{3} \tau_{0}\right) & =-h_{\mathrm{s}} \sigma_{3} \eta_{2} \tau_{3} \\
\left(K \sigma_{0} \eta_{1} \tau_{0}\right)^{-1}\left(-h_{\mathrm{s}} \sigma_{3} \eta_{2} \tau_{3}\right)\left(K \sigma_{0} \eta_{1} \tau_{0}\right) & =-h_{\mathrm{s}} \sigma_{3} \eta_{2} \tau_{3}
\end{aligned}
$$

while for the uniform field and the symmetries $\mathcal{T} \circ M_{x}$ and $\mathcal{T} \circ \tilde{M}_{y}$,

$$
\begin{aligned}
& \left(K \sigma_{1} \eta_{3} \tau_{0}\right)^{-1}\left(-h \sigma_{0} \eta_{2} \tau_{3}\right)\left(K \sigma_{1} \eta_{3} \tau_{0}\right)=-h \sigma_{0} \eta_{2} \tau_{3} \\
& \left(K \sigma_{0} \eta_{1} \tau_{0}\right)^{-1}\left(-h \sigma_{0} \eta_{2} \tau_{3}\right)\left(K \sigma_{0} \eta_{1} \tau_{0}\right)=-h \sigma_{0} \eta_{2} \tau_{3} .
\end{aligned}
$$

Since the remaining terms in the real-space triplon Hamiltonian (resulting from the bilinear spin intractions) remain invariant by the previously stated argument, the overall real-space triplon Hamiltonians with staggered and uniform Zeeman fields are invariant with respect to $M_{x}, \mathcal{T} \circ \tilde{M}_{y}$ and $\mathcal{T} \circ M_{x}, \mathcal{T} \circ \tilde{M}_{y}$, respectively.

Finally, as claimed in the main text, we see that the representations of the symmetries $M_{x}$ and $\mathcal{T} \circ \tilde{M}_{y}$ appropriate to the staggered field do indeed anticommute:

$$
\left\{\sigma_{1} \eta_{3} \tau_{0}, K \sigma_{0} \eta_{1} \tau_{0}\right\}=0
$$

Using this prescription and the transformations for the spins, it is straightforward to find the representations given in Eqs. 20 and 24.

\section{Appendix D: Effect of exchange disorder on the corner modes}

We have investigated the robustness of the corner modes against various types of disorder in finite-size samples. We find that weak disorder that preserves the symmetries on average do not destroy the corner modes. Specifically, we introduce disorder of strength $w$ by uniformly sampling the Heisenberg interactions on each interdimer bond from the interval $[J-w / 2, J+w / 2]$, where $J \in\left\{J_{1,2}^{x}, J_{1,2}^{y}\right\}$. Indeed, Fig. 5 illustrates that increasing disorder in the tBBH model increases the penetration depth of the corner states into the bulk (see first and second rows of Fig. 5), but only when the disorder is strong enough to close the bandgap do the corner modes become delocalized (see third row of Fig. 5). A similar analysis on the tSOC model yielded analogous results.
[1] W. A. Benalcazar, B. A. Bernevig, and T. L. Hughes, Science 357, 61 (2017).
[2] F. Schindler, A. M. Cook, M. G. Vergniory, Z. Wang, S. S. P. Parkin, B. A. Bernevig, and T. Neupert, Science 


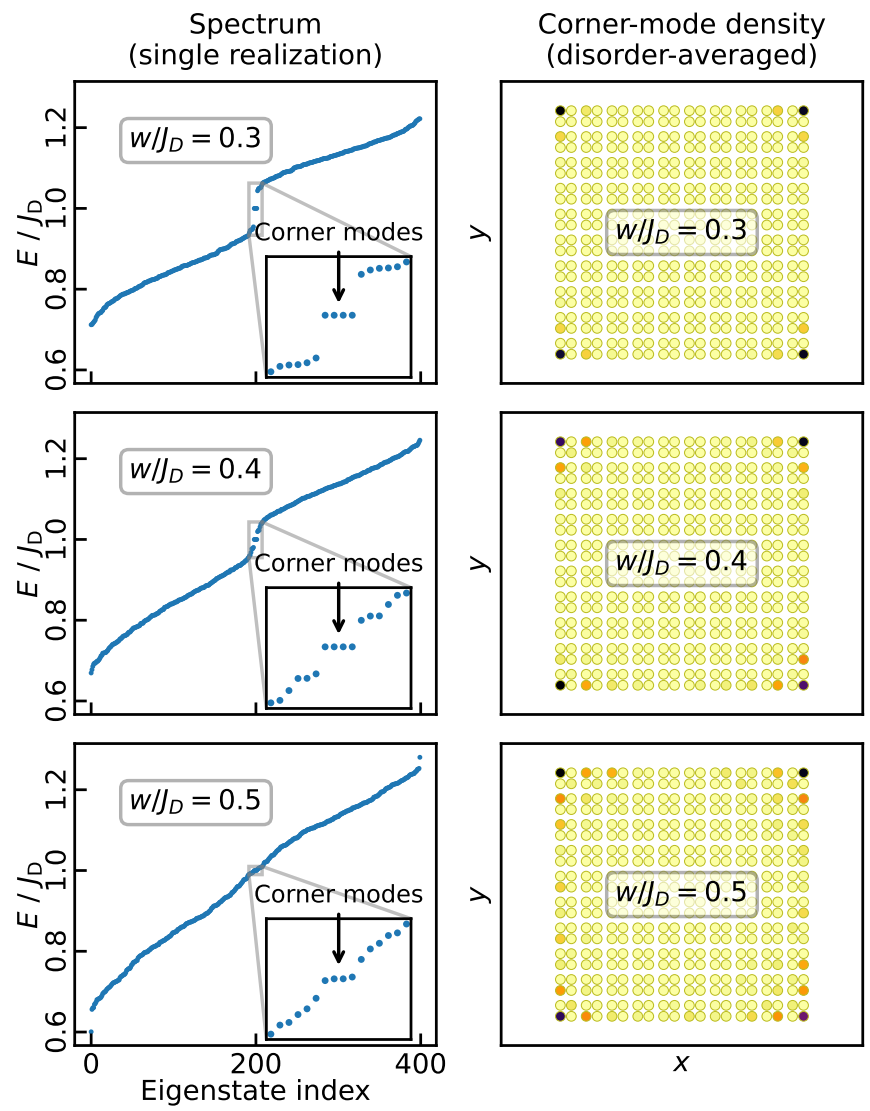

FIG. 5. Spectrum for a single disorder realization (left) and disorder-averaged total corner-mode density (right) for disorder of the form described in the main text, and for three different disorder strengths $\left(w / J_{\mathrm{D}}=0.3, w / J_{\mathrm{D}}=0.4, w / J_{\mathrm{D}}=0.5\right)$. The corner modes become delocalized once the disorder is strong enough to close the bandgap. The mean parameter values for the inter-dimer Heisenberg-interactions are $\overline{J_{1}^{x}}=\overline{J_{1}^{y}}=0.02 J_{\mathrm{D}}, \overline{J_{2}^{x}}=\overline{J_{2}^{y}}=0.2 J_{\mathrm{D}}$.

Advances 4, eaat0346 (2018).

[3] M. Serra-Garcia, V. Peri, R. Süsstrunk, O. R. Bilal, T. Larsen, L. G. Villanueva, and S. D. Huber, Nature 555, 342 (2018).

[4] S. Imhof, C. Berger, F. Bayer, J. Brehm, L. W. Molenkamp, T. Kiessling, F. Schindler, C. H. Lee, M. Greiter, T. Neupert, et al., Nature Physics 14, 925 (2018).

[5] C. W. Peterson, W. A. Benalcazar, T. L. Hughes, and G. Bahl, Nature 555, 346 (2018).

[6] A. El Hassan, F. K. Kunst, A. Moritz, G. Andler, E. J. Bergholtz, and M. Bourennane, Nature Photonics 13, 697 (2019).

[7] X.-W. Luo and C. Zhang, Phys. Rev. Lett. 123, 073601 (2019).

[8] T. Liu, J. J. He, and F. Nori, Phys. Rev. B 98, 245413 (2018).

[9] X. Zhu, Phys. Rev. B 97, 205134 (2018).

[10] K. Laubscher, D. Loss, and J. Klinovaja, Phys. Rev. Research 1, 032017 (2019).

[11] Z. Yan, F. Song, and Z. Wang, Phys. Rev. Lett. 121, 096803 (2018).
[12] Q. Wang, C.-C. Liu, Y.-M. Lu, and F. Zhang, Phys. Rev. Lett. 121, 186801 (2018).

[13] M. Kheirkhah, Y. Nagai, C. Chen, and F. Marsiglio, Phys. Rev. B 101, 104502 (2020).

[14] Z. Yan, Phys. Rev. Lett. 123, 177001 (2019).

[15] V. Dwivedi, C. Hickey, T. Eschmann, and S. Trebst, Phys. Rev. B 98, 054432 (2018).

[16] H. Wang and A. Principi, arXiv preprint arXiv:2101.01180 (2021).

[17] J. Guo, J. Sun, X. Zhu, C.-A. Li, H. Guo, and S. Feng, Journal of Physics: Condensed Matter 34, 035603 (2021).

[18] H. Watanabe, Y. Kato, H. C. Po, and Y. Motome, Phys. Rev. B 103, 134430 (2021).

[19] Y. You, T. Devakul, F. J. Burnell, and T. Neupert, Phys. Rev. B 98, 235102 (2018).

[20] O. Dubinkin and T. L. Hughes, Physical Review B 99 , 235132 (2019).

[21] A. Mook, S. A. Díaz, J. Klinovaja, and D. Loss, Phys. Rev. B 104, 024406 (2021).

[22] M. J. Park, S. Lee, and Y. B. Kim, Phys. Rev. B 104, L060401 (2021).

[23] Z. Li, Y. Cao, P. Yan, and X. Wang, npj Computational Materials 5, 1 (2019).

[24] A. Sil and A. K. Ghosh, Journal of Physics: Condensed Matter 32, 205601 (2020).

[25] T. Hirosawa, S. A. Díaz, J. Klinovaja, and D. Loss, Physical Review Letters 125, 207204 (2020).

[26] B. Sriram Shastry and B. Sutherland, Physica B+C 108 1069 (1981).

[27] R. W. Smith and D. A. Keszler, Journal of Solid State Chemistry 81, 305 (1989).

[28] Y. Sasago, K. Uchinokura, A. Zheludev, and G. Shirane, Phys. Rev. B 55, 8357 (1997).

[29] H. Tanaka, A. Oosawa, T. Kato, H. Uekusa, Y. Ohashi, K. Kakurai, and A. Hoser, Journal of the Physical Society of Japan 70, 939 (2001).

[30] H. Sakurai, N. Tsuboi, K. Yoshimura, and K. Kosuge, Journal of the Physical Society of Japan 71, 999 (2002).

[31] C. Rüegg, N. Cavadini, A. Furrer, H. U. Güdel, K. Krämer, H. Mutka, A. Wildes, K. Habicht, and P. Vorderwisch, Nature 423, 62 (2003).

[32] C. Rüegg, B. Normand, M. Matsumoto, C. Niedermayer, A. Furrer, K. W. Krämer, H.-U. Güdel, P. Bourges, Y. Sidis, and H. Mutka, Phys. Rev. Lett. 95, 267201 (2005).

[33] T. Giamarchi, C. Rüegg, and O. Tchernyshyov, Nature Physics 4, 198 (2008).

[34] S. E. Sebastian, N. Harrison, C. D. Batista, L. Balicas, M. Jaime, P. A. Sharma, N. Kawashima, and I. R. Fisher, Nature 441, 617 (2006).

[35] M. E. Zayed, C. Rüegg, J. Larrea J., A. M. Läuchli, C. Panagopoulos, S. S. Saxena, M. Ellerby, D. F. McMorrow, T. Strässle, S. Klotz, G. Hamel, R. A. Sadykov, V. Pomjakushin, M. Boehm, M. Jiménez-Ruiz, A. Schneidewind, E. Pomjakushina, M. Stingaciu, K. Conder, and H. M. Rønnow, Nature Physics 13, 962 (2017).

[36] G. Hester, H. S. Nair, T. Reeder, D. R. Yahne, T. N. DeLazzer, L. Berges, D. Ziat, J. R. Neilson, A. A. Aczel, G. Sala, J. A. Quilliam, and K. A. Ross, Phys. Rev. Lett. 123, 027201 (2019).

[37] C. K. Majumdar and D. K. Ghosh, Journal of Mathematical Physics 10, 1388 (1969).

[38] A. W. Sandvik, Phys. Rev. Lett. 98, 227202 (2007). 
[39] J. Romhányi, K. Penc, and R. Ganesh, Nature Communications 6, 6805 (2015).

[40] P. A. McClarty, F. Krüger, T. Guidi, S. Parker, K. Refson, A. Parker, D. Prabhakaran, and R. Coldea, Nature Physics 13, 736 (2017).

[41] W. P. Su, J. R. Schrieffer, and A. J. Heeger, Phys. Rev. Lett. 42, 1698 (1979).

[42] K. Nawa, K. Tanaka, N. Kurita, T. J. Sato, H. Sugiyama, H. Uekusa, S. Ohira-Kawamura, K. Nakajima, and H. Tanaka, Nature communications 10, 1 (2019).

[43] D. G. Joshi and A. P. Schnyder, Phys. Rev. B 96, 220405 (2017).

[44] S. Sachdev and R. N. Bhatt, Phys. Rev. B 41, 9323 (1990).

[45] A. Collins, C. J. Hamer, and Z. Weihong, Phys. Rev. B 74, 144414 (2006).

[46] J. Romhányi, K. Totsuka, and K. Penc, Phys. Rev. B 83, 024413 (2011).

[47] J. Colpa, Physica A: Statistical Mechanics and its Applications 93, 327 (1978).

[48] J.-P. Blaizot and G. Ripka, Quantum theory of finite systems (MIT Press, Cambridge, MA, 1986).

[49] Y. Kawaguchi and M. Ueda, Physics Reports 520, 253 (2012), spinor Bose-Einstein condensates.

[50] M. Lein and K. Sato, Phys. Rev. B 100, 075414 (2019).

[51] W. A. Benalcazar, B. A. Bernevig, and T. L. Hughes, Phys. Rev. B 96, 245115 (2017).

[52] R. Resta, Phys. Rev. Lett. 80, 1800 (1998).

[53] L. Fidkowski, T. S. Jackson, and I. Klich, Phys. Rev. Lett. 107, 036601 (2011).

[54] G. Engelhardt and T. Brandes, Phys. Rev. A 91, 053621 (2015).
[55] R. Jackiw and C. Rebbi, Phys. Rev. D 13, 3398 (1976).

[56] G. Massarelli, I. Khait, and A. Paramekanti, To appear as preprint (2021).

[57] S. Bravyi, D. P. DiVincenzo, and D. Loss, Annals of Physics 326, 2793 (2011).

[58] R. Winkler, in Spin-Orbit Coupling Effects in TwoDimensional Electron and Hole Systems (Springer, Berlin, Heidelberg, 2003) pp. 201-206.

[59] X. Zhang, F. Mahmood, M. Daum, Z. Dun, J. A. M. Paddison, N. J. Laurita, T. Hong, H. Zhou, N. P. Armitage, and M. Mourigal, Phys. Rev. X 8, 031001 (2018).

[60] P. Chauhan, F. Mahmood, H. J. Changlani, S. M. Koohpayeh, and N. P. Armitage, Phys. Rev. Lett. 124, 037203 (2020).

[61] D. G. Joshi, A. P. Schnyder, and S. Takei, Phys. Rev. B 98, 064401 (2018).

[62] P. Willke, Y. Bae, K. Yang, J. L. Lado, A. Ferrón, T. Choi, A. Ardavan, J. Fernández-Rossier, A. J. Heinrich, and C. P. Lutz, Science 362, 336 (2018).

[63] C. Du, T. van der Sar, T. X. Zhou, P. Upadhyaya, F. Casola, H. Zhang, M. C. Onbasli, C. A. Ross, R. L. Walsworth, Y. Tserkovnyak, and A. Yacoby, Science 357, 195 (2017).

[64] F. Casola, T. van der Sar, and A. Yacoby, Nature Reviews Materials 3, 17088 (2018).

[65] J. F. Rodriguez-Nieva, K. Agarwal, T. Giamarchi, B. I. Halperin, M. D. Lukin, and E. Demler, Phys. Rev. B 98, 195433 (2018).

[66] S. Chatterjee, J. F. Rodriguez-Nieva, and E. Demler, Phys. Rev. B 99, 104425 (2019). 